\title{
Symplectomorphism groups and embeddings of balls into rational ruled 4-manifolds
}

\author{
Martin Pinsonnault
}

\begin{abstract}
Let $M_{\mu}^{0}$ denote $S^{2} \times S^{2}$ endowed with a split symplectic form $\mu \sigma \oplus \sigma$ normalized so that $\mu \geqslant 1$ and $\sigma\left(S^{2}\right)=1$. Given a symplectic embedding $\iota: B_{c} \hookrightarrow M_{\mu}^{0}$ of the standard ball of capacity $c \in(0,1)$ into $M_{\mu}^{0}$, consider the corresponding symplectic blow-up $\widetilde{M}_{\mu, c}^{0}$. In this paper, we study the homotopy type of the symplectomorphism group $\operatorname{Symp}\left(\widetilde{M}_{\mu, c}^{0}\right)$ and that of the space $\Im \operatorname{Emb}\left(B_{c}, M_{\mu}^{0}\right)$ of unparametrized symplectic embeddings of $B_{c}$ into $M_{\mu}^{0}$. Writing $\ell$ for the largest integer strictly smaller than $\mu$, and $\lambda \in(0,1]$ for the difference $\mu-\ell$, we show that the symplectomorphism group of a blow-up of 'small' capacity $c<\lambda$ is homotopically equivalent to the stabilizer of a point in $\operatorname{Symp}\left(M_{\mu}^{0}\right)$, while that of a blow-up of 'large' capacity $c \geqslant \lambda$ is homotopically equivalent to the stabilizer of a point in the symplectomorphism group of a non-trivial bundle $\mathbb{C} P^{2} \# \overline{\mathbb{C}}^{2}$ obtained by blowing down $\widetilde{M}_{\mu, c}^{0}$. It follows that, for $c<\lambda$, the space $\Im \operatorname{Emb}\left(B_{c}, M_{\mu}^{0}\right)$ is homotopy equivalent to $S^{2} \times S^{2}$, while, for $c \geqslant \lambda$, it is not homotopy equivalent to any finite $\mathrm{CW}$-complex. A similar result holds for symplectic ruled manifolds diffeomorphic to $\mathbb{C} P^{2} \# \overline{\mathbb{C}}^{2}$. By contrast, we show that the embedding spaces $\Im \operatorname{Emb}\left(B_{c}, \mathbb{C} P^{2}\right)$ and $\Im \operatorname{Emb}\left(B_{c_{1}} \sqcup B_{c_{2}}, \mathbb{C} P^{2}\right)$, if non-empty, are always homotopy equivalent to the spaces of ordered configurations $F\left(\mathbb{C} P^{2}, 1\right) \simeq \mathbb{C} P^{2}$ and $F\left(\mathbb{C} P^{2}, 2\right)$. Our method relies on the theory of pseudo-holomorphic curves in 4-manifolds, on the computation of Gromov invariants in rational 4-manifolds, and on the inflation technique of Lalonde and McDuff.
\end{abstract}

\section{Introduction}

Let $(X, \omega)$ be a symplectic 4-manifold and consider the space $\operatorname{Emb}\left(B_{c}, X\right)$ of symplectic embeddings of the standard ball $B_{c} \subset \mathbb{R}^{4}$ of radius $r$ and capacity $c=\pi r^{2}$ into $X$, endowed with its natural $C^{\infty}$ topology. We define the space of unparametrized symplectic embeddings by setting

$$
\Im \operatorname{Emb}\left(B_{c}, X\right):=\operatorname{Emb}\left(B_{c}, X\right) / \operatorname{Symp}\left(B_{c}\right) .
$$

The homotopy type of this space carries important information about the diffeomorphism class of the symplectic form $\omega$. For instance, $\Im \operatorname{Emb}\left(B_{c}, X\right)$ is non-empty if and only if $c$ is smaller than the Gromov width of $(X, \omega)$, and two symplectic blow-ups of $(X, \omega)$ of same capacity $c$ are isotopic if $\Im \operatorname{Emb}\left(B_{c}, X\right)$ is connected. It is therefore natural to investigate the homotopy type of this space, at least for some amenable families of symplectic 4-manifolds.

In a previous paper with Lalonde [LP04], we proposed a general framework to study the homotopy type of $\Im \operatorname{Emb}\left(B_{c}, X\right)$ based on the correspondence between embeddings of balls and symplectic

Received 28 March 2006, revised 1 August 2007, accepted in final form 1 October 2007, published online 14 March 2008.

2000 Mathematics Subject Classification 57R17, 57S05.

Keywords: symplectomorphism groups, symplectic balls, rational ruled 4-manifolds, J-holomorphic curves.

This research was funded partly by NSERC grant BP-301203-2004.

This journal is (C) Foundation Compositio Mathematica 2008. 


\section{Pinsonnault}

blow-ups. We showed that when $\Im \operatorname{Emb}\left(B_{c}, X\right)$ is non-empty and connected, the natural action of the symplectomorphism group $\operatorname{Symp}(X, \omega)$ on $\Im \operatorname{Emb}\left(B_{c}, X\right)$ is transitive and defines a fibration

$$
\operatorname{Symp}\left(\widetilde{X}_{\iota}, \Sigma\right) \rightarrow \operatorname{Symp}(X, \omega) \rightarrow \Im \operatorname{Emb}\left(B_{c}, X\right)
$$

whose fiber over an embedding $\iota: B_{c} \hookrightarrow X$ is homotopy equivalent to the $\operatorname{subgroup} \operatorname{Symp}\left(\widetilde{X}_{\iota}, \Sigma\right)$ of symplectomorphisms of the symplectic blow-up along $\iota$ sending the exceptional divisor $\Sigma$ to itself. We used this to investigate the space of symplectic balls in the product

$$
M_{\mu}^{0}:=\left(S^{2} \times S^{2}, \mu \sigma \oplus \sigma\right),
$$

where $\sigma$ is an area form such that $\sigma\left(S^{2}\right)=1$ and where the parameter $\mu$ lies in the interval $[1,2]$. By computing the rational homotopy invariants of the symplectomorphism group of the blow-up of $M_{\mu}^{0}$ at a ball of capacity $c \in(0,1)$, and using the computation of the rational cohomology ring of $\operatorname{Symp}\left(M_{\mu}^{0}\right)$ by Abreu and McDuff [AM00], we showed that the homotopy type of the space $\Im \operatorname{Emb}\left(B_{c}, M_{\mu}^{0}\right)$ depends on the capacity of the ball $B_{c}$ in an essential way.

Theorem 1.1 (Lalonde and Pinsonnault [LP04]).

(i) If $\mu=1$, then, for any $c \in(0,1)$, the embedding space $\Im \operatorname{Emb}\left(B_{c}, M_{\mu}^{0}\right)$ is homotopy equivalent to $S^{2} \times S^{2}$.

(ii) If $\mu \in(1,2]$ and $c \in(0, \mu-1)$, then $\Im \operatorname{Emb}\left(B_{c}, M_{\mu}^{0}\right)$ is homotopy equivalent to $S^{2} \times S^{2}$.

(iii) If $\mu \in(1,2]$ and $c \in[\mu-1,1)$, then the rational homotopy groups of $\Im \operatorname{Emb}\left(B_{c}, M_{\mu}^{0}\right)$ vanish in all dimensions except in dimensions 2,3 , and 4 , in which cases we have $\pi_{2}=\mathbb{Q}^{2}, \pi_{3}=\mathbb{Q}^{3}$, and $\pi_{4}=\mathbb{Q}$. Moreover, this space is not homotopically equivalent to a finite $C W$-complex.

The main purpose of the present paper is the completion of these calculations for arbitrary rational ruled symplectic 4-manifolds, that is, for all symplectic $S^{2}$-bundles over $S^{2}$. As a byproduct, we also describe the homotopy type of $\Im \operatorname{Emb}\left(B_{c}, \mathbb{C} P^{2}\right)$ and that of the space $\Im \operatorname{Emb}\left(B_{c} \sqcup B_{c^{\prime}}, \mathbb{C} P^{2}\right)$ of two disjoint balls of capacities $c$ and $c^{\prime}$ in $\mathbb{C} P^{2}$.

\subsection{General setting and main results}

1.1.1 Symplectic birational equivalences. In order to state our results, we first review some essential facts about rational ruled symplectic 4-manifolds and their blow-ups.

To begin, recall that the classification theorem of Lalonde and McDuff [LM94] asserts that any two cohomologous symplectic forms on a ruled 4-manifold are symplectomorphic. It follows that a rational ruled symplectic 4-manifold is, after rescaling, symplectomorphic to either:

(a) the trivial bundle $M_{\mu}^{0}:=\left(S^{2} \times S^{2}, \omega_{\mu}^{0}\right)$, where the symplectic area of a section $S^{2} \times\{*\}$ is $\mu \geqslant 1$ and the area of a fiber $\{*\} \times S^{2}$ is 1 ; or

(b) the non-trivial bundle $M_{\mu}^{1}:=\left(S^{2} \tilde{\times} S^{2}, \omega_{\mu}^{1}\right)$, where the symplectic area of a section of selfintersection -1 is $\mu>0$ and the area of a fiber is 1 .

We will denote by $F^{i}$ the homology class of a fiber in $M_{\mu}^{i}, i \in\{0,1\}$, and by $B^{i}$ the class of a section of self-intersection $-i$. With this choice of normalization, the area of a fiber is less than or equal to the area of any embedded symplectic sphere of non-negative self-intersection. It follows that the Gromov width of $M_{\mu}^{i}$ is always $1=\omega_{\mu}\left(F^{i}\right)$, so that the embedding space $\Im \operatorname{Emb}\left(B_{c}, M_{\mu}^{i}\right)$ is non-empty if and only if $c \in(0,1)$.

In [McD96] McDuff showed that the symplectomorphism type of a symplectic blow-up of $M_{\mu}^{i}$ along an embedded ball of capacity $c \in(0,1)$ depends only on the capacity $c$, and not on the particular embedding used in the construction (this is often referred to as 'the uniqueness of symplectic blow-ups' for $M_{\mu}^{i}$ ). Hereafter we will write $\widetilde{M}_{\mu, c}^{i}$ for the symplectic blow-up of $M_{\mu}^{i}$ at a ball of capacity $c, \Sigma^{i}$ for the corresponding exceptional divisor, and $E^{i}=\left[\Sigma^{i}\right]$ for its homology class. 


\section{SyMPLECTOMORPHISMS AND EMBEDDINGS}

Now recall that the (smooth) blow-up of $M^{0}=S^{2} \times S^{2}$ can be identified with the blow-up of $M^{1}=S^{2} \tilde{\times} S^{2}$ via a diffeomorphism which preserves the fibers away from the exceptional loci. At the homology level, this identification induces an isomorphism $H_{2}\left(\widetilde{M}^{0} ; \mathbb{Z}\right) \simeq H_{2}\left(\widetilde{M}^{1} ; \mathbb{Z}\right)$ which acts on the classes $\left\{B^{0}, F^{0}, E^{0}\right\}$ and $\left\{B^{1}, F^{1}, E^{1}\right\}$ as follows:

$$
\begin{aligned}
F^{1} & \longleftrightarrow F^{0}, \\
B^{1}+F^{1}-E^{1} & \longleftrightarrow B^{0}, \\
F^{1}-E^{1} & \longleftrightarrow E^{0}, \\
B^{1} & \longleftrightarrow B^{0}-E^{0}, \\
E^{1} & \longleftrightarrow F^{0}-E^{0} .
\end{aligned}
$$

Note that $\widetilde{M^{0}}$ contains exactly three exceptional classes (that is, classes represented by smooth embedded spheres of self-intersection -1), namely $E^{0}, F^{0}-E^{0}$, and $B^{0}-E^{0}$. The blow-down of an exceptional curve representing either $F^{0}-E^{0}$ or $B^{0}-E^{0}$ defines a manifold diffeomorphic to the non-trivial bundle $S^{2} \tilde{\times} S^{2}$ while the blow-down of a curve in class $E^{0}$ yields $S^{2} \times S^{2}$. In particular, blowing up $S^{2} \times S^{2}$ and then blowing down along a curve representing $F^{0}-E^{0}$ defines a birational equivalence $S^{2} \times S^{2} \rightarrow S^{2} \tilde{\times} S^{2}$ which preserves the fibers.

When one considers this birational equivalence in the symplectic category, the uniqueness of symplectic blow-ups implies that the blow-up of $M_{\mu}^{0}$ at a ball of capacity $c \in(0,1)$ is symplectomorphic to the blow-up of $M_{\mu-c}^{1}$ at a ball of capacity $1-c$. Conversely, if $\mu \geqslant c$, the blow-up of $M_{\mu}^{1}$ with capacity $c \in(0,1)$ is symplectomorphic to the blow-up of $M_{1+\mu-c}^{0}$ with capacity $1-c$. For this reason, given a ruled manifold $M_{\mu}^{i}$ and a number $c \in(0,1)$ such that $c \leqslant \mu$, it is convenient to define the 'c-related' manifold $M_{\mu}^{i, c}$ by setting

$$
M_{\mu}^{i, c}:= \begin{cases}M_{\mu-c}^{1} & \text { if } i=0, \\ M_{1+\mu-c}^{0} & \text { if } i=1,\end{cases}
$$

so that we can view the rational manifold $\widetilde{M}_{\mu, c}^{i}$ either as a $c$-blow-up of $M_{\mu}^{i}$ or as a $(1-c)$-blow-up of $M_{\mu}^{i, c}$. As we will see later, this leads to a duality between symplectic balls in $M_{\mu}^{i}$ and in $M_{\mu}^{i, c}$.

In the special case of $M_{\mu}^{1}$ with $0<\mu<c<1$, the blow-up $\widetilde{M}_{\mu, c}^{1}$ is again symplectomorphic to $\widetilde{M}_{1+\mu-c, 1-c}^{0}$. However, the base class $B$ now has smaller area than the fiber class $F$, so that our choice of normalization forces us to interchange the two $S^{2}$ factors in the product $S^{2} \times S^{2}$ and to rescale the symplectic form. On the blow-up manifold, this induces a diffeomorphism which swaps the exceptional classes $B^{1}=B^{0}-E^{0}$ and $E^{1}=F^{0}-E^{0}$. It follows that $\widetilde{M}_{\mu, c}^{1}$ is conformally symplectomorphic to $\widetilde{M}_{\mu^{\prime}, c^{\prime}}^{0}$ where

$$
\mu^{\prime}:=\frac{1}{1+\mu-c} \geqslant 1 \quad \text { and } \quad c^{\prime}:=\frac{1-c}{1+\mu-c} \in(0,1) .
$$

Of course, this lack of symmetry will be reflected in the structure of $\Im \operatorname{Emb}\left(B_{c}, M_{\mu}^{i}\right)$ when $0<\mu<$ $c<1$.

1.1.2 Symplectomorphism groups of $\widetilde{M}_{\mu, c}^{i}$. The greater part of this paper is devoted to the determination of the homotopy type of the symplectomorphism groups $\operatorname{Symp}\left(\widetilde{M}_{\mu, c}^{i}\right)$ and $\operatorname{Symp}\left(\widetilde{M}_{\mu, c}^{i}, \Sigma\right)$. As in the works of Gromov [Gro85], Abreu [Abr98], and Abreu and McDuff [AM00], our understanding comes from a study of the natural action of these groups on the contractible space $\mathcal{J}_{\mu, c}^{i}$ of tamed almost complex structures on $\widetilde{M}_{\mu, c}^{i}$. This analysis was initiated in [LP04] where we showed that this action preserves a stratification of $\mathcal{J}_{\mu, c}^{i}$ whose strata are indexed by certain configurations 


\section{Pinsonnault}

of $J$-holomorphic curves or, equivalently, by equivalence classes of toric structures (that is, conjugacy classes of 2-tori $\left.T^{2} \subset \operatorname{Symp}\left(\widetilde{M}_{\mu, c}^{i}\right)\right)$. For instance, if we consider the blow-up of $M_{\mu}^{i}$ at a ball of capacity $c \leqslant \mu$, and if we write $\mu=\ell+\lambda$ with $\ell \in \mathbb{N}$ and $\ell<\mu \leqslant \ell+1$, the number $N$ of toric structures on $\widetilde{M}_{\mu, c}^{i}$ is

$$
N= \begin{cases}2 \ell+1+i & \text { if } c<\lambda, \\ 2 \ell+i & \text { if } \lambda \leqslant c,\end{cases}
$$

so that the geometry of the stratification changes precisely when $\ell$ crosses an integer or when $c$ crosses the critical number $\lambda$.

The analysis of $J$-holomorphic curves in $\widetilde{M}_{\mu, c}^{i}$ is carried further in $\S 2$ of the present paper. This is used in $\S 3$ to study how the homotopy types of the groups $\operatorname{Symp}\left(\widetilde{M}_{\mu, c}^{i}\right)$ and $\operatorname{Symp}\left(\widetilde{M}_{\mu, c}^{i}, \Sigma\right)$ change as the parameters $\mu$ and $c$ vary. We first note that, in most cases, the distinction between $\operatorname{Symp}\left(\widetilde{M}_{\mu, c}^{i}\right)$ and its subgroup $\operatorname{Symp}\left(\widetilde{M}_{\mu, c}^{i}, \Sigma\right)$ is immaterial.

Lemma 1.2. Given $c \leqslant \mu$, let $S$ be an embedded symplectic sphere in $\widetilde{M}_{\mu, c}^{i}$ representing one of the exceptional classes $E^{i}$ or $F^{i}-E^{i}$. Then $\operatorname{Symp}\left(\widetilde{M}_{\mu, c}^{i}\right)$ is homotopy equivalent to its subgroup $\operatorname{Symp}\left(\widetilde{M}_{\mu, c}^{i}, S\right)$.

Our main result states that, assuming $c \leqslant \mu$, the homotopy type of $\operatorname{Symp}\left(\widetilde{M}_{\mu, c}^{i}, \Sigma\right)$ changes only when $\ell$ or $c$ crosses a critical number, that is, when the set of toric structures on $\widetilde{M}_{\mu, c}^{i}$ changes. This is the content of the following 'stability' theorem.

Theorem 1.3. Fix $i \in\{0,1\}$. Let $\mu_{1} \geqslant 1-i$ and write $\mu_{1}=\ell+\lambda_{1}$ with $\ell \in \mathbb{N}$ and $\lambda_{1} \in(0,1]$. Similarly, given a number $\mu_{2} \geqslant 1-i$ in the interval $(\ell, \ell+1]$, write $\mu_{2}=\ell+\lambda_{2}$ with $\lambda_{2} \in(0,1]$. Consider $c_{1}, c_{2} \in(0,1)$ such that either $c_{1}<\lambda_{1}$ and $c_{2}<\lambda_{2}$, or $\lambda_{1} \leqslant c_{1}$ and $\lambda_{2} \leqslant c_{2}$. Suppose also that $c_{i} \leqslant \mu_{i}$. Then the symplectomorphism groups $\operatorname{Symp}\left(\widetilde{M}_{\mu_{1}, c_{1}}^{i}, \Sigma\right)$ and $\operatorname{Symp}\left(\widetilde{M}_{\mu_{2}, c_{2}}^{i}, \Sigma\right)$ are homotopy equivalent.

Observe that from the homotopy fibration (1) above, it follows that the behavior of symplectic balls of capacity $c \leqslant \mu$ in $M_{\mu}^{i}$ depends only on whether $c<\lambda$ or $\lambda \leqslant c$. This motivates the following definition.

Definition 1.4. Write $\mu=\ell+\lambda$ with $\ell \in \mathbb{N}$ and $\ell<\mu \leqslant \ell+1$. We say that an embedded symplectic ball $B_{c} \hookrightarrow M_{\mu}^{i}$ is small if its capacity $c$ is strictly less than $\lambda$. It is said to be big if $\lambda \leqslant c$.

It is easy to see that, if $c \leqslant \mu$, the blow-up of a 'small' ball $B_{c}$ in $M_{\mu}^{i}$ is symplectomorphic to the blow-up of a 'big' ball in the $c$-related manifold $M_{\mu}^{i, c}$. Conversely, blowing up a 'big' ball in $M_{\mu}^{i}$ is equivalent to blowing up a 'small' ball in $M_{\mu}^{i, c}$. Assuming $c<\lambda$ and looking at the limit $c \rightarrow 0$ (while keeping $\mu$ constant), we obtain the following simple homotopy-theoretic description of the $\operatorname{groups} \operatorname{Symp}\left(\widetilde{M}_{\mu, c}^{i}, \Sigma\right) \simeq \operatorname{Symp}\left(\widetilde{M}_{\mu, c}^{i}\right)$.

TheOREM 1.5. Let $\widetilde{M}_{\mu, c}^{i}$ be the symplectic blow-up of $M_{\mu}^{i}$ at a ball of capacity $c \in(0,1)$. In the case $i=1$, suppose also that $c \leqslant \mu$. Write $\mu=\ell+\lambda$ where $\ell$ is the unique integer such that $\ell<\mu \leqslant \ell+1$ and where $\lambda \in(0,1]$. Then, the following hold.

(i) When $c<\lambda$, the symplectomorphism group of the blow-up manifold $\widetilde{M}_{\mu, c}^{i}$ is homotopy equivalent to the group $\operatorname{Symp}\left(M_{\mu}^{i}, p\right)$ of symplectomorphisms that fix a point $p$ in $M_{\mu}^{i}$.

(ii) When $\lambda \leqslant c$, the symplectomorphism group of the blow-up manifold $\widetilde{M}_{\mu, c}^{i}$ is homotopy equivalent to the group $\operatorname{Symp}\left(M_{\mu}^{i, c}, p\right)$ of symplectomorphisms that fix a point $p$ in the c-related manifold $M_{\mu}^{i, c}$. 


\section{SyMPLECTOMORPHISMS AND EMBEDDINGS}

In the case $0<\mu<c<1$, the symplectomorphism group $\operatorname{Symp}\left(\widetilde{M}_{\mu, c}^{1}\right)$ is homeomorphic to the group $\operatorname{Symp}\left(\widetilde{M}_{\mu^{\prime}, c^{\prime}}^{0}\right)$ where $\mu^{\prime}:=c /(1+\mu-c)$ and $c^{\prime}:=\mu /(1+\mu-c)$. However, the groups $\operatorname{Symp}\left(\widetilde{M}_{\mu, c}^{1}\right)$ and $\operatorname{Symp}\left(\widetilde{M}_{\mu, c}^{1}, \Sigma\right)$ are no longer homotopy equivalent. Nevertheless, the techniques used in the proof of Theorem 1.3 apply mutatis mutandis and yield the following simple result.

Theorem 1.6. When $0<\mu<c<1$, the symplectomorphism group $\operatorname{Symp}\left(\widetilde{M}_{\mu, c}^{1}, \Sigma\right)$ is homotopy equivalent to the stabilizer of a point in $M_{\mu}^{1}$.

From the homotopy equivalences of Theorems 1.5 and 1.6, and from the computations of the rational homotopy invariants of $\operatorname{Symp}\left(M_{\mu}^{i}\right)$ by Abreu and $\operatorname{McDuff}$ [AM00], we easily obtain a description of the rational homotopy module $\pi_{*}\left(\operatorname{Symp}\left(\widetilde{M}_{\mu, c}^{i}\right)\right) \otimes \mathbb{Q}$, of the cohomology ring $H^{*}\left(\operatorname{Symp}\left(\widetilde{M}_{\mu, c}^{i}\right) ; \mathbb{Q}\right)$, of the module $H^{*}\left(\operatorname{BSymp}\left(\widetilde{M}_{\mu, c}^{i}\right) ; \mathbb{Q}\right)$, and of the Pontrjagin and Samelson products in $H_{*}\left(\operatorname{Symp}\left(\widetilde{M}_{\mu, c}^{i}\right) ; \mathbb{Q}\right)$ and $\pi_{*}\left(\operatorname{BSymp}\left(\widetilde{M}_{\mu, c}^{i}\right)\right) \otimes \mathbb{Q}$; see $\S 3.5$.

1.1.3 Spaces of symplectic embeddings. Our understanding of the symplectomorphism groups of $\widetilde{M}_{\mu, c}^{i}$ can now be applied to the study of the embedding space $\Im \operatorname{Emb}\left(B_{c}, M_{\mu}^{i}\right)$ via the homotopy fibration (1). In the case $c \leqslant \mu$, this gives the following result.

Theorem 1.7. Given a rational ruled manifold $M_{\mu}^{i}$, write $\mu=\ell+\lambda$ where $\ell$ is the unique integer such that $\ell<\mu \leqslant \ell+1$ and where $\lambda \in(0,1]$. Let $c \in(0,1)$. In the case $i=1$, suppose also that $c \leqslant \mu$. Then, the following hold.

(i) When $c<\lambda$, the space $\Im \operatorname{Emb}\left(B_{c}, M_{\mu}^{i}\right)$ is homotopy equivalent to $M_{\mu}^{i}$, that is, small symplectic balls in $M_{\mu}^{i}$ behave like points.

(ii) When $\lambda \leqslant c$, the rational homotopy of the $\operatorname{space} \Im \operatorname{Emb}\left(B_{c}, M_{\mu}^{i}\right)$ is generated, additively, by two elements of degree 2 , two elements of degree 3 , one element of degree $4 \ell-1+2 i$, and one element of degree $4 \ell+2 i$.

In the case $0<\mu<c<1$, we obtain an even simpler result.

TheOREM 1.8. If $0<\mu<c<1$, the space $\Im \operatorname{Emb}\left(B_{c}, M_{\mu}^{1}\right)$ is homotopy equivalent to $M_{\mu}^{1}$.

Corollary 1.9. For all $\mu \in(0,1]$ and $c \in(0,1), \Im \operatorname{Emb}\left(B_{c}, M_{\mu}^{1}\right)$ is homotopy equivalent to $M_{\mu}^{1}$.

The same techniques can be applied to investigate the homotopy-theoretic properties of other embedding spaces. In particular, our computations allow us to describe the space $\Im \operatorname{Emb}\left(B_{c} ; \mathbb{C} P^{2}\right)$ and the space of symplectic embeddings $B_{c_{1}} \sqcup B_{c_{2}} \hookrightarrow \mathbb{C} P^{2}$ of two disjoint balls in $\mathbb{C} P^{2}$.

Theorem 1.10. Consider $\mathbb{C} P^{2}$ endowed with its standard Fubini-Study symplectic form normalized so that the area of a line is 1 . Then, the following hold.

(i) For any $c \in(0,1)$, the space $\Im \operatorname{Emb}\left(B_{c}, \mathbb{C} P^{2}\right)$ is homotopy equivalent to $\mathbb{C} P^{2}$.

(ii) Given $c_{1}, c_{2} \in(0,1)$ so that $c_{1}+c_{2}<1$, the space $\Im \operatorname{Emb}\left(B_{c_{1}} \sqcup B_{c_{2}} ; \mathbb{C} P^{2}\right)$ is homotopy equivalent to the space $F\left(2, \mathbb{C} P^{2}\right)$ of ordered configurations of two points in $\mathbb{C} P^{2}$.

We conclude this introduction with some comments on possible generalizations of our results. Following Anjos and Granja [AG04] and Abreu, Granja and Kitchloo [AGK06], the action of the symplectomorphism group $\operatorname{Symp}\left(\widetilde{M}_{\mu, c}^{i}\right)$ on the natural stratification of the space of almost complex structures $\mathcal{J}_{\mu, c}^{i}$ will lead to a homotopy decomposition of $\operatorname{BSymp}\left(\widetilde{M}_{\mu, c}^{i}\right)$ as a homotopy colimit over the poset category of toric structures and their intersections. In turn, this should yield a better description of the homotopy type of the embedding space $\Im \operatorname{Emb}\left(B_{c}, M_{\mu}^{i}\right)$ in the case of 'big' balls. In another direction, we note that if we impose certain conditions on the capacities, it is possible to 


\section{Pinsonnault}

get a partial understanding of the space $\Im \operatorname{Emb}\left(B_{c_{1}} \sqcup \cdots \sqcup B_{c_{r}}, \mathbb{C} P^{2}\right)$ of $r \geqslant 3$ disjoint symplectic balls in $\mathbb{C} P^{2}$ by proving versions of the stability theorem (Theorem 1.3). In these manifolds, the structure of pseudo-holomorphic curves can be quite complicated and is best understood through the action of the group of birational equivalences of $X_{r}$. But new phenomena also occur which make the analysis of the symplectomorphism groups more difficult. For instance, a proper understanding of the action of $\operatorname{Symp}\left(X_{r}\right)$ on the contractible space $\mathcal{J}\left(X_{r}\right)$ requires a description of various moduli spaces of complex structures, and this may no longer yield a computable description of the homotopy type of $\operatorname{Symp}\left(X_{r}\right)$. Another problem is that the symplectomorphism groups $\operatorname{Symp}\left(X_{c_{1}, \ldots, c_{r}}\right)$ are not necessarily connected. Indeed, Seidel showed that for the monotone blow-ups $X_{r}, 5 \leqslant r \leqslant 8$, the group $G=\operatorname{Symp}\left(X_{r}\right) \cap \operatorname{Diff}_{0}$ is not connected; see [Sei03]. His proof relies on the action of $\pi_{0}(G)$ on the Donaldson quantum category of $X_{r}$ and yields computable invariants only in the monotone case. However, it seems possible to combine our method with that of Seidel in order to get new insights on the homotopy type of $\operatorname{Symp}\left(X_{r}\right)$ in the non-monotone case. These ideas will be pursued elsewhere.

1.1.4 Conventions. We endow all diffeomorphism groups with the $C^{\infty}$ topology. A theorem of Milnor [Mil59] implies that these topological groups are homotopy equivalent to finite or countable $\mathrm{CW}$-complexes. Consequently, we do not distinguish between weak homotopy equivalences and genuine homotopy equivalences. Note also that our notation differs slightly from the one used in [AM00] where the manifold $M_{\lambda}^{i}$ corresponds to our $M_{\mu}^{i}$ with $\mu=1+\lambda$. Finally, since the present paper is a continuation of [LP04], we refer the reader to that paper for the details of several arguments and proofs.

\section{Structure of $J$-holomorphic curves in $\widetilde{M}_{\mu, c}^{i}$}

In this section, we describe the structure of $J$-holomorphic spheres representing special homology classes in the blow-up $\widetilde{M}_{\mu, c}^{i}$. This analysis relies on well-known results about $J$-holomorphic curves in symplectic 4-manifolds that we briefly recall for convenience. The proofs can be found in [AL94, Gro85, HLS97, MS94].

\section{1 $J$-holomorphic curves in symplectic 4-manifolds}

A parametrized $J$-sphere is a map $u: \mathbb{C} P^{1} \rightarrow M$ which satisfies the Cauchy-Riemann equation $d u \circ j=J \circ d u$. We always assume that $u$ is somewhere injective, that is, there is a point $z \in \mathbb{C} P^{1}$ such that $d u_{z} \neq 0$ and $z=u^{-1}(u(z))$. In this case, $u$ is not a multiple cover and its image $S=u\left(\mathbb{C} P^{2}\right)$ represents a homology class in $H_{2}(X ; \mathbb{Z})$ that we denote $[S]$ or $[u]$.

An almost complex structure, $J$, is tamed by a symplectic form $\omega$ if $\omega(v, J v)>0$ for all $v \neq 0$. Note that if $J \in \mathcal{J}$ then every $J$-holomorphic submanifold $C \subset M$ is symplectic and, conversely, every symplectic submanifold of $(M, \omega)$ is $J$-holomorphic for some $J \in \mathcal{J}$. In general, if a class $A \neq 0$ can be represented by a $J$-holomorphic sphere, for some $J \in \mathcal{J}(\omega)$, then $\omega(A)>0$. For any symplectic manifold, the space of tamed almost complex structures, denoted by $\mathcal{J}=\mathcal{J}(M, \omega)$, is always non-empty and contractible. It follows that the first Chern class $c_{1}(T M)$ is independent of the choice of $J \in \mathcal{J}$.

Given a class $A \in H_{2}(X ; \mathbb{Z})$, we define the universal moduli space $\mathcal{M}_{p}(A, \mathcal{J})$ of parametrized holomorphic $A$-spheres by setting:

$$
\mathcal{M}_{p}(A, \mathcal{J})=\{(u, J): J \in \mathcal{J}, u \text { is } J \text {-holomorphic, and }[u]=A\} .
$$

A fundamental result of the theory of $J$-holomorphic curves asserts that $\mathcal{M}_{p}(A, \mathcal{J})$ is always a Fréchet manifold and that the projection $P_{A}: \mathcal{M}_{p}(A, \mathcal{J}) \rightarrow \mathcal{J}$ is a Fredholm map of index $2 c_{1}(A)+4$. 


\section{SyMPLECTOMORPHISMS AND EMBEDDINGS}

Given $J \in \mathcal{J}$, the preimage $\mathcal{M}_{p}(A, J)=P_{A}^{-1}(J)$ is the space of all parametrized $J$-holomorphic $A$ spheres. The automorphism group $G=\operatorname{PSL}(2, \mathbb{C})$ of $\mathbb{C} P^{1}$ acts freely on $\mathcal{M}_{p}(A, J)$ by reparametrization and the quotient $\mathcal{M}(A, J)=\mathcal{M}_{p}(A, J) / G$ is the moduli space of unparametrized $J$-holomorphic $A$-spheres. An almost complex structure, $J \in \mathcal{J}$, is said to be regular for the class $A$ if it is a regular value for the projection $P_{A}$. In this case, the sets $\mathcal{M}_{p}(A, J)$ and $\mathcal{M}(A, J)$ are smooth manifolds of dimensions $2 c_{1}(A)+4$ and $2 c_{1}(A)-2$ respectively. The set $J_{\text {reg }}$ of all such regular $J$ is a subset of second category in $\mathcal{J}$.

Although the moduli space $\mathcal{M}_{p}(A, J)$ is never compact, its quotient $\mathcal{M}(A, J)=\mathcal{M}_{p}(A, J) / G$ can be compactified by adding cusp-curves, that is, connected unions of possibly multiply covered $J$-curves. ${ }^{1}$ Indeed we have the following theorem.

TheOREM (Gromov's compactness theorem). If a sequence $\left\{J_{i}\right\} \subset \mathcal{J}$ converges to $J_{\infty} \in \mathcal{J}$ and if $S_{i}$ are unparametrized $J_{i}$-holomorphic spheres of bounded symplectic area, then there is a subsequence of the $S_{i}$ which converges weakly to a genuine $J$-sphere or to a $J$-holomorphic cusp-curve.

A crucial fact is that weak convergence implies convergence in the Hausdorff topology of subsets. Consequently, the area of the limit is the limit of the areas of the converging subsequence. In particular, if all the $S_{i}$ represent the same class $A$, and if the sequence $\left\{S_{i}\right\}$ converges to a cuspcurve $S_{\infty}$ made of several components $C_{1} \cup \cdots \cup C_{k}$, each having multiplicity $m_{j}>0$, then their homology classes $m_{j}\left[C_{j}\right]$ provide a decomposition of $A$ with $\omega(A)>\omega\left(C_{j}\right)>0$.

In dimension $4, J$-holomorphic spheres have a number of special properties which often reduce geometric questions to homological ones. These are as follows:

Regularity. The Hofer-Lizan-Sikorav regularity criterion asserts that, if $c_{1}(A) \geqslant 1$, then all $J \in \mathcal{J}$ are regular values of the projection $P_{A}$.

Positivity of intersections. Two distinct $J$-spheres $S_{1}$ and $S_{2}$ in a 4 -manifold have only a finite number of intersection points. Each such point contributes positively to the algebraic intersection number $S_{1} \cdot S_{2}$. Moreover, $S_{1} \cdot S_{2}=0$ if and only if the spheres are disjoint, and $S_{1} \cdot S_{2}=1$ if and only if they meet exactly once transversally.

Adjunction formula. Given a symplectic 4-manifold $M$ and a class $A \in H_{2}(M ; \mathbb{Z})$, we define the virtual genus of $A$ by the formula

$$
g_{v}(A)=1+\frac{1}{2}\left(A \cdot A-c_{1}(A)\right)
$$

If $A$ is represented by the image of a somewhere injective $J$-holomorphic map $u: \mathbb{C} P^{1} \rightarrow M$, then $g_{v}(A)$ is a non-negative integer which is zero if and only if $u$ is an embedding.

Exceptional curves (see [McD90, Lemma 3.1]). Let $A \in H_{2}(M ; \mathbb{Z})$ be a homology class which is represented by an embedded symplectic sphere $S$, and such that $A \cdot A=-1$. Then the space $\mathcal{J}_{A}$ of all $J \in \mathcal{J}$ for which the class $A$ is represented by an embedded $J$-sphere contains an open, dense, and path-connected subspace of $\mathcal{J}$.

\subsection{Gromov invariants}

We now recall some facts from Taubes-Seiberg-Witten theory and Gromov invariants. Given a four-dimensional symplectic manifold $(X, \omega)$ and a class $A \in H_{2}(X ; \mathbb{Z})$ such that $k(A)=\frac{1}{2}(A \cdot A+$ $\left.c_{1}(A)\right) \geqslant 0$, the Gromov invariant of $A$ as defined by Taubes ${ }^{2}$ in [Tau96] counts, for a generic almost

\footnotetext{
${ }^{1}$ Although the compactification of the moduli space is best described using the finer notions of stable maps and stable curves, the simpler notion of cusp-curves is sufficient for our purpose.

${ }^{2}$ The original definition given by Taubes must be modified slightly to handle multiply covered curves and to have an equivalence between Gromov invariants and Seiberg-Witten invariants. See [LL99] for a complete discussion.
} 


\section{Pinsonnault}

complex structure $J$ tamed by $\omega$, the algebraic number of embedded $J$-holomorphic curves in class $A$ passing through $k(A)$ generic points. In general, these curves may be disconnected and, in that case, components of non-negative self-intersection are embedded holomorphic curves of some genus $g \geqslant 0$, while all other components are exceptional spheres. An important corollary of Gromov's compactness theorem is that, given a tamed $J$, any class $A$ with non-zero Gromov invariant $\operatorname{Gr}_{\omega}(A)$ is represented by a collection of $J$-holomorphic curves or cusp-curves.

The Gromov invariants only depend on the deformation class of $\omega$, that is, $\operatorname{Gr}_{\omega_{t}}(A)$ is constant along any one-parameter family of symplectic forms $\omega_{t}$. In fact, in dimension 4 , it follows from the work of Taubes that Gromov invariants are also smooth invariants of $M$.

Now suppose that $(X, \omega)$ is some blow-up of a rational ruled manifold $M_{\mu}^{i}$ with canonical homology class $K=-\mathrm{PD}\left(c_{1}\right)$. Since $b_{2}^{+}=1$, the wall-crossing formula of $\mathrm{Li}$ and Liu for SeibergWitten invariants (see [LL95, Corollary 1.4]) implies that for every class $A \in H_{2}(X, \mathbb{Z})$ verifying the condition $k(A)=\frac{1}{2}\left(A \cdot A+c_{1}(A)\right) \geqslant 0$ we have

$$
\operatorname{Gr}(A) \pm \operatorname{Gr}(K-A)= \pm 1 .
$$

In particular, if $\operatorname{Gr}(K-A)=0$ (for example, when $\omega(K-A) \leqslant 0$ ), then $\operatorname{Gr}(A) \neq 0$.

\subsection{Structure of $J$-holomorphic curves in $\widetilde{M}_{\mu, c}^{i}$}

As before, we write $\mu=\ell+\lambda$ with $\ell \in \mathbb{N}$ and $\lambda \in(0,1]$. Since we can always obtain $\widetilde{M}_{\mu, c}^{i}$ by blowing up a ball in the product $S^{2} \times S^{2}$, we will work only with $M_{\mu}^{0}$ and, to simplify notation, we will omit the superscript ' 0 ' on homology classes in $H_{2}\left(\widetilde{M}_{\mu, c}^{0}, \mathbb{Z}\right)$. In particular, we will write $B$ for $\left[S^{2} \times\{*\}\right]$, $F$ for $\left[\{*\} \times S^{2}\right]$, and $E$ for the class of the exceptional divisor in $\widetilde{M}_{\mu, c}^{0}$.

Lemma 2.1. Given $\mu \geqslant 1, c \in(0,1)$, if a class $A=p B+q F-r E \in H_{2}\left(M_{\mu}^{0}, \mathbb{Z}\right)$ has a simple $J$-holomorphic representative, then $p \geqslant 0$. Moreover, if $p=0$, then $A$ is either $F, F-E$ or $E$. Furthermore, if $p=1$, then $r \in\{0,1\}$.

Proof. This follows easily from the adjunction inequality $0 \leqslant 2 g_{v}(A)=2(p-1)(q-1)-r(r-1)$, the positivity of the symplectic area $\omega(A)=\mu p+q-c r$, and our choice of normalization: $\mu=\omega(B) \geqslant$ $\omega(F)=1>\omega(E)=c>0$.

Lemma 2.2. Given $\mu \geqslant 1, c \in(0,1)$, and any tamed almost complex structure $J \in \mathcal{J}_{\mu, c}$, each of the exceptional classes $E$ and $F-E$ are represented by a unique embedded $J$-holomorphic curve. Consequently, if a class $A=p B+q F-r E$ has a simple $J$-holomorphic representative, then $p \geqslant r \geqslant 0$, unless $A \in\{E, F-E\}$.

Proof. We know that the set of almost complex structures $J$ for which the exceptional classes have embedded $J$-holomorphic representatives is open and dense. Moreover, by positivity of intersections, any such $J$-holomorphic representative is necessarily unique. Thus, by Gromov's compactness theorem, we only need to show that these classes cannot be represented by multiply covered curves nor by cusp-curves.

First, consider the class $E$. Since $E$ is a primitive class, it cannot be represented by a multiply covered curve. Suppose it is represented by a cusp curve $C=\bigcup_{1}^{N} m_{i} C_{i}$, where $N \geqslant 2$ and each component $C_{i}$ is simple. By Lemma 2.1, the homology class of each component $C_{i}$ is either $F, E$ or $F-E$. The cases $\left[C_{i}\right]=F$ and $\left[C_{i}\right]=E$ are impossible since the symplectic area of each component must be strictly less than the area of $E$. But then each component $C_{i}$ represents the class $F-E$, which is also impossible. Therefore, the class $E$ cannot be represented by a cusp-curve.

The same arguments apply to the class $F-E$ and show that it is always represented by an embedded $J$-holomorphic sphere. Consequently, if a class $A=p B+q F-r E$ has a simple 


\section{SyMPLECTOMORPHISMS AND EMBEDDINGS}

$J$-holomorphic representative, the positivity of intersections implies that $0 \leqslant A \cdot(F-E)=p-r$ and $0 \leqslant A \cdot E=r$, unless $A$ is either $E$ or $F-E$.

Lemma 2.3. Given $\mu \geqslant 1, c \in(0,1)$, and a tamed almost complex structure $J \in \mathcal{J}_{\mu, c}$, the moduli space $\mathcal{M}(F, J)$ of embedded $J$-holomorphic spheres representing the fiber class $F$ is a non-empty, open, two-dimensional manifold.

Proof. Since the class $F$ is regular $\left(c_{1}(F)=2 \geqslant 1\right)$, the dimension of the space of unparametrized $J$-holomorphic representatives is $2 c_{1}(F)-2=2$ whenever it is non-empty. But since $\operatorname{Gr}(F)=1$, this space is non-empty for a generic tamed almost complex structure $J$, and there is exactly one embedded $J$-holomorphic representative of $F$ passing through a $k(F)=\frac{1}{2}\left(c_{1}(F)+F^{2}\right)=1$ generic point of $\widetilde{M}_{\mu, c}^{0}$.

Now suppose that for some tamed $J$, there is no embedded $J$-holomorphic curve representing the class $F$. By Gromov's compactness theorem, a generic point of $\widetilde{M}_{\mu, c}^{0}$ must belong to either a multiply covered curve or a cusp-curve in class $F$. Being simple, the class $F$ cannot be represented by a multiply covered curve. If it is represented by a $J$-holomorphic cusp-curve, the two previous lemmas imply that this cusp-curve is necessarily of the form $(F-E) \cup E$ so that it cannot contain a generic point. We conclude that $\mathcal{M}(F, J)$ is non-empty for any tamed almost complex structure $J$.

Let us define the classes $D_{i} \in H_{2}\left(\widetilde{M}_{\mu, c}^{0}, \mathbb{Z}\right), i \in \mathbb{Z}$, by setting $D_{2 k+1}=B+k F$ and $D_{2 k}=$ $B+k F-E$. Note that we have

$$
D_{i} \cdot D_{j}=\left\lfloor\frac{i+j}{2}\right\rfloor,
$$

where $\lfloor x\rfloor$ denotes the greatest integer strictly less than $x$. In particular, $D_{i} \cdot D_{i}=i-1$ and the adjunction formula implies that any $J$-holomorphic sphere in class $D_{i}$ must be embedded. Note also that the virtual dimension of the spaces of unparametrized $J$-curves representing $D_{i}$ is $2 i$ and that $k\left(D_{i}\right)=i$.

Lemma 2.4. Given an integer $i \geqslant 1$, the Gromov invariant of the class $D_{i}$ is non-zero. Consequently, for a generic tamed almost complex structure $J$, there exists an embedded J-holomorphic sphere representing $D_{i}$ and passing through a generic set of $k\left(D_{i}\right)=i$ points in $\widetilde{M}_{\mu, c}^{0}$.

Proof. Let $K=-2(B+F)-E$ be the Poincaré dual of the canonical class of $\widetilde{M}_{\mu, c}^{0}$. Because $\omega\left(K-D_{i}\right) \leqslant 0$, we know that $\operatorname{Gr}\left(K-D_{i}\right)=0$, and since $k=k\left(D_{i}\right) \geqslant 0$, formula (4) implies that $\operatorname{Gr}\left(D_{i}\right)= \pm 1$. Given a generic $J$, the class $D_{i}$ is thus represented by the disjoint union of some embedded $J$-holomorphic curve of genus $g \geqslant 0$ with some exceptional $J$-spheres. But since $D_{i}$ has non-negative intersection with the three exceptional classes $B-E, F-E$, and $E$, this representative is in fact connected and its genus is given by the adjunction formula.

Lemma 2.5. The set of tamed almost complex structures on $\widetilde{M}_{\mu, c}^{0}$ for which the class $D_{-1}=B-E$ is represented by an embedded $J$-holomorphic sphere is open and dense in $\mathcal{J}_{\mu, c}$. If for a given $J$ there is no such sphere, then there is a unique integer $1 \leqslant m \leqslant \ell$ such that either the class $D_{-2 m}=B-m F$ or the class $D_{-2 m-1}=B-m F-E$ is represented by a unique embedded J-holomorphic sphere.

Proof. Since the class $B-E$ is exceptional, the set of almost complex structures $J$ for which it has an embedded $J$-holomorphic representative is open and dense. Being primitive, it has no multiply covered representative. Suppose it is represented by a cusp curve $C=\bigcup_{1}^{N} m_{i} C_{i}$, where $N \geqslant 2$. We must show that one of its component is a representative of $B-m F$ or $B-m F-E$ for some integer $1 \leqslant m \leqslant \ell$. By Lemmas 2.1 and 2.2 , any cusp-curve gives a decomposition of the class $B-E$ of the form

$$
D_{i}=(B+m F-r E)+\sum_{a} s_{a} F+t(F-E)+u E,
$$




\section{Pinsonnault}

where the multiplicities $s_{a}, t$, and $u$ are all positive and where $r \in\{0,1\}$. This implies that $m<0$. Since the symplectic area of the component in class $B+m F-r E$ must be strictly positive, $|m|$ must be less than or equal to $\ell$. Note that if $|m|<\ell$, the class $B-|m| F-E$ has positive area since $c<1$, while if $|m|=\ell$, it has positive area only if $c<\lambda$. Finally, since the intersection of $B-|m| F-|r| E$ with any other class $D_{i}, i \leqslant 0$, is negative, at most one such class can be represented by a $J$-holomorphic curve for a given tamed almost complex structure $J$.

Let $\tilde{\mathcal{J}}_{\mu, c, m}$ be the space of all almost complex structure $J \in \tilde{\mathcal{J}}_{\mu, c}$ such that the class $D_{-m}$ is represented by an embedded $J$-holomorphic sphere. Lemma 2.5 shows that the space $\tilde{\mathcal{J}}_{\mu, c}$ is the disjoint union of its subspaces $\tilde{\mathcal{J}}_{\mu, c, m}$, that is,

$$
\tilde{\mathcal{J}}_{\mu, c}=\tilde{\mathcal{J}}_{\mu, c, 0} \sqcup \cdots \sqcup \tilde{\mathcal{J}}_{\mu, c, N} \quad \text { where } N= \begin{cases}2 \ell & \text { if } c<\lambda, \\ 2 \ell-1 & \text { if } \lambda \leqslant c .\end{cases}
$$

Lemma 2.6. Given a tamed almost complex structure $J$ belonging to the strata $\tilde{\mathcal{J}}_{\mu, c, m}^{0}$, a class $D_{i}$, $i \neq-m$, is represented by some embedded $J$-holomorphic sphere if and only if $D_{i} \cdot D_{-m} \geqslant 0$. In particular, if either $c<\lambda$ and $i \geqslant 2 \ell+1$, or $\lambda \leqslant c$ and $i \geqslant 2 \ell$, then, for all $J \in \tilde{\mathcal{J}}_{\mu, c}$, the class $D_{i}$ has embedded $J$-holomorphic representatives.

Proof. We first note that the presence of a $J$-holomorphic curve in class $D_{-m}$ forbids the existence of an embedded representative of any class $D_{i}, i \neq-m$, such that $D_{i} \cdot D_{-m}<0$. Now given any $J \in$ $\tilde{\mathcal{J}}_{\mu, c, m}$, consider a class $D_{i}$ such that $D_{i} \cdot D_{-m} \geqslant 0$ and suppose that $D_{i}$ does not have any embedded $J$-holomorphic representative. Note that we have necessarily $i \geqslant 1$ and hence $\operatorname{Gr}\left(D_{i}\right) \neq 0$. Since $D_{i}$ is generically represented by embedded holomorphic spheres, there must be a $J$-holomorphic cusp-curve representing $D_{i}$ and passing through $k=k\left(D_{i}\right)=i$ generic points $\left\{p_{1}, \ldots, p_{k}\right\}$. Again, Lemmas 2.1 and 2.2 imply that any such $J$-holomorphic cusp-curve $C=\bigcup_{1}^{N} m_{i} C_{i}$ with at least two components must define a decomposition of $D_{i}$ of the form

$$
D_{i}=D_{r}+\sum_{a} s_{a} F+\sum_{b} t_{b}(F-E)+\sum_{c} u_{c} E,
$$

where the multiplicities $s_{a}, t_{b}$, and $u_{c}$ are all positive and where $D_{r} \cdot D_{-m} \geqslant 0$ unless $r=-m$.

Consider the reduced cusp-curve $\bar{C}$ obtained from $C$ by removing all but one copy of its repeated components, and by replacing multiply covered components by their underlying simple curve. The curves $\bar{C}$ and $C$ have the same image and hence contain the same subset of special points in $\left\{p_{1}, \ldots, p_{k}\right\}$. The cusp-curve $\bar{C}$ represents a class of the form

$$
[\bar{C}]=D_{r}+\sum_{a} F+\sum_{b}(F-E)+\sum_{c} E
$$

since, by the adjunction formula, there are no simple curves representing $m F, m(F-E)$, or $m E$ for $m \geqslant 2$. When $r \geqslant 0$, all the components are regular and the dimension of the space of unparametrized reduced cusp-curves of this type is simply

$$
\operatorname{dim} \mathcal{M}(\bar{C}, J)=\sum_{\text {components } \bar{C}_{i}} \operatorname{dim} \mathcal{M}\left(\left[\bar{C}_{i}\right], J\right)=2 c_{1}(\bar{C})-2 N,
$$

where $N$ is the number of components of $\bar{C}$. Since $c_{1}(\bar{C}) \leqslant c_{1}\left(D_{i}\right)$, the dimension of unparametrized and reduced cusp-curves of type $\bar{C}$ is less than $2 k\left(D_{i}\right)$ and this implies that no such cusp-curve can pass through $k\left(D_{i}\right)$ generic points.

When $r<0$, the only possibility is $r=-m$. The component in class $D_{-m}$ has negative selfintersection and is thus isolated. Therefore, the dimension of the space of cusp-curves of type $\bar{C}$ is at most $2 n$, where $n$ is the number of components representing the class $F$. Note that this number 


\section{SyMPLECTOMORPHISMS AND EMBEDDINGS}

$n$ is maximal when the reduced cusp-curve is of one of the three following forms:

$$
D_{i}=D_{-m}+\sum F, \quad D_{i}=D_{-m}+\sum F+(F-E), \quad \text { or } \quad D_{i}=D_{-m}+\sum F+E .
$$

In each case the dimension of the moduli space of cusp-curves is strictly less than $2 k\left(D_{i}\right)=2 i$ and, again, this implies that no cusp-curve of type $\bar{C}$ can pass through $k\left(D_{i}\right)$ generic points.

The last statement of the lemma follows from the fact that, when $c<\lambda$, the highest codimensional strata in $\tilde{\mathcal{J}}_{\mu, c}$ corresponds to structures for which the class $D_{-2 \ell}=B-\ell F-E$ is represented, and $D_{-2 \ell} \cdot D_{i} \geqslant 0$ if and only if $i \geqslant 2 \ell+1$. Similarly, when $\lambda \leqslant c$, the last strata corresponds to curves $D_{-2 \ell+1}=B-\ell F$ and $D_{-2 \ell+1} \cdot D_{i} \geqslant 0$ if and only if $i \geqslant 2 \ell$.

Remark 2.7. Our identification of $\tilde{M}_{\mu+1-c, 1-c}^{0}$ with $\tilde{M}_{\mu, c}^{1}$ implies that when we blow up $M_{\mu}^{1}$ with capacity $0<\mu<c<1$, the exceptional classes that are always represented by embedded $J$-spheres are $B^{1}$ and $F^{1}-E^{1}$. In particular, the class $E^{1}$ of the exceptional divisor can be represented by $J$-holomorphic cusp-curves. This will force us to study the space $\Im \operatorname{Emb}\left(B_{c}, M_{\mu}^{1}\right), 0<\mu<c<1$, separately; see $\S 4$.

\subsection{The stratification of $\tilde{\mathcal{J}}_{\mu, c}$}

Adapting the gluing techniques used in McDuff [McD00], one can show that the decomposition (5) is a genuine stratification. In order to state its main properties, we now briefly recall the projective realizations of the ruled manifolds $M_{\mu}^{i}$ as Hirzebruch surfaces.

For any $\mu>0$ and any integer $i \geqslant 0$ satisfying $\mu-i / 2>0$, let $\mathbb{C} P^{1} \times \mathbb{C} P^{2}$ be endowed with the Kähler form $(\mu-a(i)) \sigma_{1}+\sigma_{2}$ where $\sigma_{j}$ is the Fubini-Study form on $\mathbb{C} P^{j}$ normalized so that the area of a linear $\mathbb{C} P^{1}$ is equal to 1 , and where $a(i)$ is $i / 2$ when $i$ is even or $(i-1) / 2$ when $i$ is odd. Let $W_{i}$ be the Hirzebruch surface defined by setting

$$
W_{i}=\left\{\left(\left[z_{0}, z_{1}\right],\left[w_{0}, w_{1}, w_{2}\right]\right) \in \mathbb{C} P^{1} \times \mathbb{C} P^{2} \mid z_{0}^{i} w_{1}=z_{1}^{i} w_{0}\right\} .
$$

The restriction of the projection $\pi_{1}: \mathbb{C} P^{1} \times \mathbb{C} P^{2} \rightarrow \mathbb{C} P^{1}$ to $W_{i}$ endows $W_{i}$ with the structure of a $\mathbb{C} P^{1}$-bundle over $\mathbb{C} P^{1}$ which is topologically $S^{2} \times S^{2}$ if $i$ is even, and $\mathbb{C} P^{2} \# \overline{\mathbb{C P}}^{2}$ if $i$ is odd. In this correspondence, the fibers are preserved and the zero section of this bundle

$$
s_{0}=\left\{\left(\left[z_{0}, z_{1}\right],[0,0,1]\right)\right\}
$$

corresponds to a section of self-intersection $-i$ which represents the class $B^{0}-\frac{1}{2} i F^{0}$ if $i$ is even or the class $B^{1}-\frac{1}{2}(i-1) F^{1}$ if $i$ is odd. Similarly, the section at infinity

$$
s_{\infty}=\left\{\left(\left[z_{0}, z_{1}\right],\left[z_{0}^{i}, z_{1}^{i}, 1\right]\right)\right\}
$$

has self-intersection $+i$ and represents $B^{0}+\frac{1}{2} i F^{0}$ or $B^{1}+\frac{1}{2}(i-1) F^{1}$ depending on the parity of $i$ (compare with (2)). By the classification theorem of ruled symplectic 4-manifolds, this correspondence establishes a symplectomorphism between $W_{i}$ and $M_{\mu}^{0}$ for all even $i$ and between $W_{i}$ and $M_{\mu}^{1}$ for all odd $i$.

Proposition 2.8 (see $[\mathrm{LP} 04, \S 4]$ and $[\mathrm{McD} 00]$ ). Let $1 \leqslant m \leqslant N$. Then the following hold.

(i) The subspace $\tilde{\mathcal{J}}_{\mu, c, m}$ is a smooth, co-oriented, codimension $2 m$ submanifold whose closure is the union $\bigsqcup_{m \leqslant i \leqslant N} \tilde{\mathcal{J}}_{\mu, c, i}$.

(ii) The subspace $\tilde{\mathcal{J}}_{\mu, c, m}$ contains a complex structure $\tilde{J}_{m}$ coming from the blow-up of the Hirzebruch surface $W_{m}$ at a point $p$ belonging to the zero section $s_{0}$.

(iii) The group $\operatorname{Symp}_{h}\left(\widetilde{M}_{\mu, c}^{0}\right)$ of symplectomorphisms acting trivially on homology acts smoothly on $\tilde{\mathcal{J}}_{\mu, c, m}$. The stabilizer of $\tilde{J}_{m}$ is the 2-torus $\tilde{T}_{m}$ which is generated by the lifts of the Kählerian isometries of $W_{m}$ fixing $p$. 


\section{Pinsonnault}

Moreover, the decomposition $\tilde{\mathcal{J}}_{\mu, c, 0} \sqcup \cdots \sqcup \tilde{\mathcal{J}}_{\mu, c, N}$ is a genuine stratification. Each $\tilde{\mathcal{J}}_{\mu, c, m}$ has a neighborhood $\mathcal{N}_{m} \subset \tilde{\mathcal{J}}_{\mu, c}$ which, once given the induced stratification, has the structure of a locally trivial fiber bundle whose typical fiber is a cone over a finite-dimensional stratified space called the link of $\tilde{\mathcal{J}}_{\mu, c . m}$ in $\tilde{\mathcal{J}}_{\mu, c}$. Finally, the link $\mathcal{L}_{m+1}$ of $\tilde{\mathcal{J}}_{\mu, c, m+1}$ in $\tilde{\mathcal{J}}_{\mu, c, m}$ is a circle.

Using the classifications of Hamiltonian $S^{1}$ - and $T^{2}$-actions on symplectic 4-manifolds given by Karshon [Kar99] and Delzant [Del88], one can show that the set of strata is in bijection with the set of conjugacy classes of maximal tori $\tilde{T}_{i}$ in $\operatorname{Symp}\left(\tilde{M}_{\mu, c}^{i}\right)$. Finally, note that the number of strata, and hence the number of inequivalent $T^{2}$-actions, changes exactly when $\mu$ crosses an integer or when the capacity $c$ reaches the critical value $\lambda$.

\section{Homotopy type of symplectomorphism groups}

\subsection{Connexity of $\operatorname{Symp}\left(\widetilde{M}_{\mu, c}^{i}\right)$ and $\operatorname{Symp}\left(\widetilde{M}_{\mu, c}^{i}, \Sigma\right)$}

Let $\operatorname{Symp}_{h}\left(\widetilde{M}_{\mu, c}^{i}\right)$ be the group of symplectomorphisms of $\widetilde{M}_{\mu, c}^{i}$ acting trivially in homology. We begin this section by recalling the main result of [LP04, §4.1].

Proposition 3.1 (see [LP04, §4.1]). In the stratification (5), the stratum $\tilde{\mathcal{J}}_{\mu, c, m}$ is homotopy equivalent to the homogeneous space $\operatorname{Symp}_{h}\left(\widetilde{M}_{\mu, c}^{0}\right) / \tilde{T}_{m}$.

The proof of this proposition is done in three steps. First, one shows that the stratum $\tilde{\mathcal{J}}_{\mu, c, m}$ is homotopy equivalent to the space $\mathcal{C}_{m}$ of all configurations of three symplectic embedded surfaces in $\widetilde{M}_{\mu, c}^{0}$ which intersect transversally and positively, and lie in classes $E, F-E$, and $D_{-m}$. Second, one proves that the configuration space $\mathcal{C}_{m}$ retracts onto the subspace $\mathcal{C}_{m}^{0}$ of configurations made of surfaces intersecting orthogonally. Finally, one shows that the symplectomorphism group $\operatorname{Symp}_{h}\left(\widetilde{M}_{\mu, c}^{0}\right)$ acts transitively on $\mathcal{C}_{m}^{0}$ and that the stabilizer of a standard configuration $\Gamma_{0}$ retracts onto the 2-torus $\tilde{T}_{m}$ of Kählerian isometries of the Hirzebruch complex structure $\tilde{J}_{m}$. Note that this last step is rather subtle since it depends on the existence of a globally contracting Liouville vector field on the complement $\widetilde{M}_{\mu, c}^{0} \backslash \Gamma_{0}$, and on Gromov's result on the contractibility of the group of compactly supported symplectomorphisms of the standard ball $B^{4} \in \mathbb{R}^{4}$.

Lemma 3.2 (see [LP04, Proposition 4.15]). The group $\operatorname{Symp}_{h}\left(\widetilde{M}_{\mu, c}^{i}\right)$ is connected.

Proof. We can suppose that $i=0$. The open stratum $\tilde{\mathcal{J}}_{\mu, c, 0}$ in the stratification (5) is path-connected and, by Proposition 2.8, is homotopy equivalent to the homogeneous space $\operatorname{Symp}_{h}\left(\widetilde{M}_{\mu, c}^{0}\right) / \tilde{T}_{0}$.

Corollary 3.3. The full group $\operatorname{Symp}\left(\widetilde{M}_{\mu, c}^{i}\right)$ is connected except for $\widetilde{M}_{1, c}^{0} \simeq \widetilde{M}_{1-c, 1-c}^{1}$ in which case it has exactly two components.

Proof. The last term in the short exact sequence

$$
1 \rightarrow \operatorname{Symp}_{h}\left(\widetilde{M}_{\mu, c}^{i}\right) \rightarrow \operatorname{Symp}\left(\widetilde{M}_{\mu, c}^{i}\right) \rightarrow \operatorname{Aut}_{c_{1}, \omega}\left(H_{2}\left(\widetilde{M}_{\mu, c}^{i} ; \mathbb{Z}\right)\right) \rightarrow 1
$$

is isomorphic to $\mathbb{Z}_{2}$ when $\omega(F-E)=\omega(B-E)$ and is trivial otherwise.

Proof of Lemma 1.2. Suppose $c \leqslant \mu$. According to Lemma 2.2, given any tame $J \in \mathcal{J}\left(\widetilde{M}_{\mu, c}^{i}\right)$, the exceptional class $[S]$ is always represented by a unique embedded $J$-holomorphic sphere. It follows that the space $\mathcal{C}[S]$ of all embedded symplectic spheres in class $[S]$ is homotopy equivalent to $\mathcal{J}\left(\widetilde{M}_{\mu, c}^{i}\right)$ so that the fibration

$$
\operatorname{Symp}_{h}\left(\widetilde{M}_{\mu, c}^{i}, S\right) \rightarrow \operatorname{Symp}_{h}\left(\widetilde{M}_{\mu, c}^{i}\right) \rightarrow \mathcal{C}[S]
$$

has a contractible base. 


\section{SyMPLECTOMORPHISMS AND EMBEDDINGS}

\subsection{Inflation and the stability theorem}

The goal of this section is to prove Theorem 1.3 which states that when $\mu_{j} \geqslant c_{j}, j \in\{1,2\}$, the symplectomorphism groups $\operatorname{Symp}\left(\tilde{M}_{\mu_{1}, c_{1}}^{i}\right)$ and $\operatorname{Symp}\left(\tilde{M}_{\mu_{2}, c_{2}}^{i}\right)$ have the same homotopy type whenever $\mu_{2} \in\left(\ell_{1}, \ell_{1}+1\right]$, and either $c_{j}<\lambda_{j}$ or $\lambda_{j} \leqslant c_{j}$. In other words, when we vary the cohomology class of $\tilde{\omega}_{\mu, c}^{i}$, the homotopy type of $\operatorname{Symp}\left(\tilde{M}_{\mu, c}^{i}\right)$ jumps exactly when a change occurs in the geometry of the corresponding stratification $\tilde{\mathcal{J}}_{\mu, c}=\tilde{\mathcal{J}}_{\mu, c, 0} \sqcup \cdots \sqcup \tilde{\mathcal{J}}_{\mu, c, N}$. Again, we will work only with $M_{\mu}^{0}$ and we will omit the superscript ' 0 ' on associated objects.

Let $G_{\mu}=\operatorname{Symp}_{h}\left(M_{\mu}^{0}\right)$ and denote by $G_{\mu, c}$ the group of symplectomorphisms of the blowup $\widetilde{M}_{\mu, c}^{0}$ acting trivially on homology. Consider the natural action of the identity component of the diffeomorphism group of $\widetilde{M}_{\mu, c}^{0}$ on the space $\mathcal{S}_{\mu, c}$ of symplectic forms isotopic to the blown-up form $\omega_{\mu, c}$. By Moser's theorem, the evaluation map ev : $\phi \mapsto \phi^{*}\left(\omega_{\mu, c}\right)$ defines a fibration

$$
G_{\mu, c}=\operatorname{Symp}_{h}\left(\widetilde{M}_{\mu, c}^{0}\right) \cap \operatorname{Diff}_{0} \rightarrow \operatorname{Diff}_{0} \stackrel{\text { ev }}{\rightarrow} \mathcal{S}_{\mu, c} .
$$

Therefore, to prove that the symplectomorphism groups $G_{\mu_{1}, c_{1}}^{i}$ and $G_{\mu_{2}, c_{2}}^{i}$ are homotopy equivalent, it would be enough to construct a homotopy equivalence between the spaces $\mathcal{S}_{\mu_{1}, c_{1}}$ and $\mathcal{S}_{\mu_{2}, c_{2}}$ such that the diagram

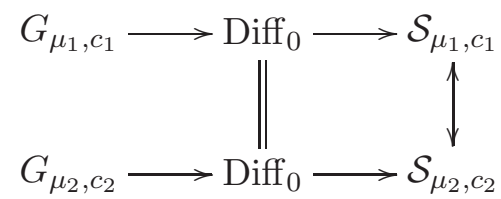

commutes up to homotopy. Since we do not know any way of constructing such a map directly, we will proceed a little differently. Indeed, we will use a nice idea of McDuff that consists in considering the action of Diff 0 on the larger space $\mathcal{X}_{\mu_{i}, c_{i}}$ of pairs $(\omega, J)$ such that $\omega \in \mathcal{S}_{\mu_{i}, c_{i}}$ and $J$ is tamed by $\omega$. This space projects to $\mathcal{S}_{\mu_{i}, c_{i}}$ and to the space $\mathcal{A}_{\mu_{i}, c_{i}}$ of almost complex structures tamed by some element of $\mathcal{S}_{\mu_{i}, c_{i}}$. It turns out that these projections are Serre fibrations with contractible fibers. This implies that the three spaces $\mathcal{S}_{\mu_{i}, c_{i}}, \mathcal{X}_{\mu_{i}, c_{i}}$, and $\mathcal{A}_{\mu_{i}, c_{i}}$ are homotopy equivalent. Moreover, the previous fibrations factorize through the spaces $\mathcal{X}_{\mu_{1}, c_{1}}$ and $\mathcal{X}_{\mu_{2}, c_{2}}$ as in the following diagram.

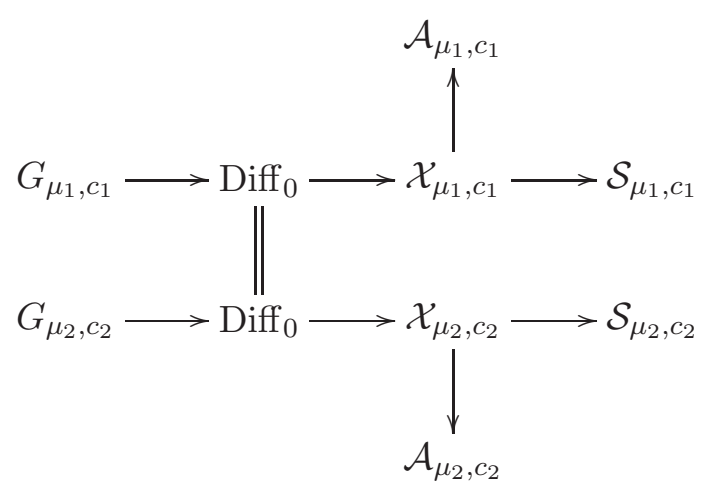

Therefore, to show that the groups $G_{\mu_{1}, c_{1}}$ and $G_{\mu_{2}, c_{2}}$ are homotopy equivalent, it is sufficient to find a homotopy equivalence $\mathcal{A}_{\mu_{1}, c_{1}} \rightarrow \mathcal{A}_{\mu_{2}, c_{2}}$ that commutes, up to homotopy, with the action of Diff $_{0}$. Indeed, we prove a stronger statement, namely the following.

Proposition 3.4. Let $\mu_{1}=\ell+\lambda_{1} \geqslant 1$. Given $\mu_{2} \in(\ell, \ell+1]$, write $\mu_{2}=\ell+\lambda_{2}$. Consider $c_{1}, c_{2} \in(0,1)$ such that either $c_{1}<\lambda_{1}$ and $c_{2}<\lambda_{2}$, or $\lambda_{1} \leqslant c_{1}$ and $\lambda_{2} \leqslant c_{2}$. Then the spaces $\mathcal{A}_{\mu_{1}, c_{1}}$ and $\mathcal{A}_{\mu_{2}, c_{2}}$ are the same.

The proof is based on the inflation lemma of Lalonde and McDuff which asserts that, in the presence of a $J$-holomorphic submanifold with non-negative self-intersection, it is possible to 


\section{Pinsonnault}

deform a symplectic form $\omega_{0}$ through a family of forms $\omega_{t}$ tamed by the almost complex structure $J$.

Proposition (Inflation lemma, see [Lal94, McD03]). Let $J \in \mathcal{J}\left(\omega_{0}\right)$ be a tame almost complex structure on a symplectic 4-manifold $\left(M, \omega_{0}\right)$ that admits a $J$-holomorphic curve $Z$ with $Z \cdot Z \geqslant 0$. Then there is a family $\omega_{t}, t \geqslant 0$, of symplectic forms that all tame $J$ and have cohomology class $\left[\omega_{t}\right]=\left[\omega_{0}\right]+t \operatorname{PD}(Z)$, where $\operatorname{PD}(Z)$ denotes the Poincaré dual of the homology class $[Z]$.

Proof. See Lemma 3.1 in [McD03].

Proof of Proposition 3.4. The proof is done in two steps. We first prove that for $\mu=\ell+\lambda \geqslant 1$ and $c<\lambda$ (respectively $\lambda \leqslant c$ ), the spaces $\mathcal{A}_{\mu, c}$ and $\mathcal{A}_{\mu^{\prime}, c}$ are the same, for all $\mu^{\prime} \in(\ell+c, \mu]$ (respectively $\left.\mu^{\prime} \in(\ell, \mu]\right)$. In the second step, we show that $\mathcal{A}_{\mu, c}=\mathcal{A}_{\mu, c^{\prime}}$ whenever the two parameters $c$ and $c^{\prime}$ verify $c \leqslant c^{\prime}<\lambda$ or $\lambda \leqslant c \leqslant c^{\prime}$.

Step 1 . We begin by showing that for any $\mu \geqslant 1$ and any $\epsilon>0$, the space $\mathcal{A}_{\mu, c}$ is included in $\mathcal{A}_{\mu+\epsilon, c}$. Indeed, Lemma 2.3 implies that for all $\left(\omega_{\mu, c}, J\right) \in \mathcal{X}_{\mu, c}$, the fiber class $F$ is represented by some embedded $J$-holomorphic sphere $S$. Therefore, it is always possible to inflate the form $\omega_{\mu, c}$ along $S$ to get a one-parameter family of symplectic forms in classes $\left[\omega_{\mu, c}\right]+\epsilon \operatorname{PD}(F)=\omega_{\mu+\epsilon, c}$. Since all these forms tame $J$, this proves that $\mathcal{A}_{\mu, c} \subset \mathcal{A}_{\mu+\epsilon, c}$.

We next show that for $\mu=\ell+\lambda \geqslant 1$ and $c<\lambda$, the space $\mathcal{A}_{\mu, c}$ in included in $\mathcal{A}_{\mu^{\prime}, c}$ whenever $\mu^{\prime} \in(\ell+c, \mu]$. This is done by inflating $\omega_{\mu, c}$ along curves representing the classes $D_{2 \ell+1}=B+\ell F$ and $D_{2 \ell+2}=B+(\ell+1) F-E$. Note that when $c<\lambda$, Lemma 2.6 implies that these two classes are always represented by embedded $J$-holomorphic spheres. Writing $d_{i}$ for an appropriate 2 -form representing $\operatorname{PD}\left(D_{i}\right)$, we define $\omega_{a b}$ for $a, b \geqslant 0$ by

$$
\omega_{a b}=\frac{\left(\omega_{\mu, c}+a d_{2 \ell+1}+b d_{2 \ell+2}\right)}{1+a+b} .
$$

We then have $\omega_{a b}(F)=1, \omega_{a b}(B)=(\mu+a \ell+b(\ell+1)) /(1+a+b)$, and $\omega_{a b}(E)=(c+b) /(1+a+b)$. The area of the exceptional class $E$ can be made equal to $c$ by setting $b=a c /(1-c) \geqslant 0$. This gives us a one-parameter family of forms $\omega_{a}$ verifying $\omega_{a}(F)=1, \omega_{a}(B)=((1-c) \mu+(\ell+c) a) /(1-c+a)$, and $\omega_{a}(E)=c$. Letting $a \rightarrow \infty$, this shows that the almost complex structure $J$ is tamed by a symplectic form in class $\left[\omega_{\ell+c+\epsilon, c}\right]$, for all $0<\epsilon \leqslant \lambda-c$. Therefore $\mathcal{A}_{\mu, c} \subset \mathcal{A}_{\mu^{\prime}, c}$, for all $\mu^{\prime} \in$ $(\ell+c, \mu]$.

Now, let us prove that for $\mu=\ell+\lambda \geqslant 1$ and $\lambda \leqslant c$, the space $\mathcal{A}_{\mu, c}$ in included in $\mathcal{A}_{\mu^{\prime}, c}$ whenever $\mu^{\prime} \in(\ell, \mu]$. Since we assume that $\lambda \leqslant c$, the classes $D_{2 \ell}$ and $D_{2 \ell+1}$ are both represented by embedded $J$-holomorphic spheres, for any choice of $\omega$-tamed $J$. By inflating $\omega_{\mu, c}$ along these curves, we get a two-parameter family of symplectic forms $\omega_{a b}=\left(\omega_{\mu, c}+a d_{2 \ell+1}+b d_{2 \ell}\right) /(1+a+b)$ for which $\omega_{a b}(F)=1, \omega_{a b}(B)=(\mu+a \ell+b \ell) /(1+a+b)$, and $\omega_{a b}(E)=(c+b) /(1+a+b)$. Again, we make the area of the exceptional class $E$ equal to $c$ by setting $b=a c /(1-c) \geqslant 0$. We then get a one-parameter family of forms $\omega_{a}$ verifying $\omega_{a}(F)=1, \omega_{a}(B)=((1-c) \mu+$ $a \ell) /(1-c+a)$, and $\omega_{a}(E)=c$. Letting $a \rightarrow \infty$, this shows that $\mathcal{A}_{\mu, c} \subset \mathcal{A}_{\mu^{\prime}, c}$ for all $\mu^{\prime} \in$ $(\ell, \mu]$.

Step 2. Let us show that $\mathcal{A}_{\mu, c^{\prime}} \subset \mathcal{A}_{\mu, c}$ whenever $c<c^{\prime}$. Given $J \in \mathcal{A}_{\mu, c^{\prime}}$, this amounts to constructing a symplectic form that tames $J$ and for which $B, F$ and $E$ have area $\mu, 1$ and $c$ respectively. By Lemmas 2.6 and 2.3, there exist embedded $J$-holomorphic spheres representing the classes $D_{2 \ell+1}=$ $B+\ell F$ and $F$. The inflation lemma implies that the Poincaré duals of $D_{2 \ell+1}$ and $F$ are represented by 2 -forms $d_{2 \ell+1}$ and $f$ such that the form $\omega_{a b}=\left(\omega_{\mu, c^{\prime}}+a d_{2 \ell+1}+b f\right) /(1+a)$ is symplectic for all $a, b \geqslant 0$. We then have $\omega_{a b}(F)=1$ and $\omega_{a b}(B)=(\mu+a \ell+b) /(1+a)$. Setting $b=$ $(\mu-\ell) a=\lambda a \geqslant 0$, the resulting family of symplectic forms $\omega_{a}$ verify $\omega_{a}(F)=1, \omega_{a}(B)=\mu$, 


\section{SYMPLECTOMORPHISMS AND EMBEDDINGS}

and $\omega_{a}(E)=c^{\prime} /(1+a)$. Since $a$ can take any non negative value, this implies that $J \in \mathcal{A}_{\mu, c}$, for all $c<c^{\prime}$.

We now suppose that $0<c<\lambda$ and prove that given any real number $c^{\prime} \in(c, \lambda)$, the space $\mathcal{A}_{\mu, c}$ is included in $\mathcal{A}_{\mu, c^{\prime}}$. This is done by inflating along embedded $J$-holomorphic spheres in classes $D_{2 \ell+2}=B+(\ell+1) F-E$ and $D_{2 \ell+1}=B+\ell F$. Indeed, setting $\omega_{a b}=\left(\omega_{\mu, c}+a d_{2 \ell+2}+b d_{2 \ell+1}\right) /(1+a+b)$ where $b=(\ell+1-\mu) /(\mu-\ell) \geqslant 0$, the resulting one-parameter family of symplectic forms verify $\omega_{a}(F)=1, \omega_{a}(B)=\mu$, and $\omega_{a}(E)=\lambda(a+c) /(\lambda+a)$. This shows that any almost complex structure $J \in \mathcal{A}_{\mu, c}$ also belongs to $\mathcal{A}_{\mu, c^{\prime}}$.

Finally, given $\lambda \leqslant c<c^{\prime}$, we need to prove that $\mathcal{A}_{\mu, c} \subset \mathcal{A}_{\mu, c^{\prime}}$. Since $\lambda \leqslant c$, Lemma 2.6 implies that the classes $D_{2 \ell}=B+\ell F-E$ and $F$ are both represented by embedded $J$-holomorphic spheres, for all $J$. We define $\omega_{a b}=\left(\omega_{\mu, c}+a d_{2 \ell}+b f\right) /(1+a)$ and set $b=a(\mu-\ell) \geqslant 0$ to get symplectic forms $\omega_{a}, a \geqslant 0$, such that $\omega_{a}(B)=\mu, \omega_{a}(F)=1$, and $\omega_{a}(E)=(a+c) /(a+1)$. Therefore, $J \in \mathcal{A}_{\mu, c^{\prime}}$, for all $\lambda \leqslant c<c^{\prime}<1$. This completes the proof of Proposition 3.4 .

Remark 3.5. Proposition 3.4 is optimal in the sense that it cannot be improved by performing the inflation process along curves representing other classes.

We can now prove the stability theorem.

Proof of Theorem 1.3. Consider the diagram (8). By Proposition 3.4, we know that the spaces $\mathcal{A}_{\mu, c}$ and $\mathcal{A}_{\mu, c^{\prime}}$ are equal whenever $c<c^{\prime}<\lambda$ or $\lambda \leqslant c<c^{\prime}$. Therefore, the spaces of symplectic forms $\mathcal{S}_{\mu, c}$ and $\mathcal{S}_{\mu, c^{\prime}}$ are homotopy equivalent and this implies that the group $\widetilde{G}_{\mu, c}$ has the same homotopy type as $\tilde{G}_{\mu, c^{\prime}}$. Finally, in the special case $\mu=1$, the full symplectomorphism group of the blow-up is a $\mathbb{Z}_{2}$-extension of its identity component and is homotopy equivalent to $T^{2} \times \mathbb{Z}_{2}$ for all $0<c<1$.

We note that the stratification $\tilde{\mathcal{J}}_{\mu, c}=\tilde{\mathcal{J}}_{\mu, c, 0} \sqcup \cdots \sqcup \tilde{\mathcal{J}}_{\mu, c, N}$ induces similar decompositions of the spaces $\mathcal{X}_{\mu, c}$ and $\mathcal{A}_{\mu, c}$ into Fréchet submanifolds invariant under the action of Diff. The proof of Proposition 3.4 shows that, for all $\mu \geqslant 1, c \in(0,1)$, and $\epsilon>0$, we have an inclusion $\mathcal{A}_{\mu, c} \hookrightarrow \mathcal{A}_{\mu+\epsilon, c}$. Moreover, if we increase $\mu$ while keeping $c$ constant, we see that the space $\mathcal{A}_{\mu, c}$ changes by the addition of a stratum of codimension $4 n-2$ each time $\mu$ crosses an integer $n$, or by the addition of a stratum of codimension $4 n$ each time $\mu$ crosses a real number of the form $n+c$. Consequently, the inclusion $\mathcal{A}_{\mu, c} \hookrightarrow \mathcal{A}_{\mu+\epsilon, c}$ is $(4 \ell-2)$-connected if $c \geqslant \lambda$, and $4 \ell$-connected if $c<\lambda$. At the level of symplectomorphism groups, the homotopy commuting diagram (8) implies that the family of inclusions $\mathcal{A}_{\mu, c} \hookrightarrow \mathcal{A}_{\mu+\epsilon, c}$ induces homotopy coherent diagrams

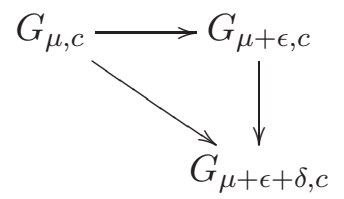

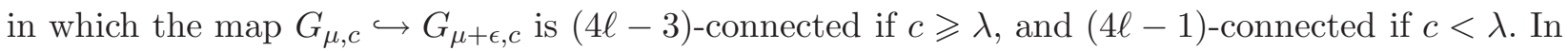
particular, we have the following result.

Proposition 3.6. For each $c \in(0,1)$ and $\epsilon \in(0, c)$, the map $G_{1+\epsilon, c} \rightarrow G_{\mu, c}$ induces an isomorphism of fundamental groups.

\subsection{Homotopy equivalences with stabilizer subgroups}

We now prove Theorem 1.5 which states that the $\operatorname{symplectomorphism} \operatorname{group} \operatorname{Symp}\left(\widetilde{M}_{\mu, c}^{0}\right)$ is homotopy equivalent to the stabilizer of a point in an associated ruled manifold. 


\section{Pinsonnault}

Proof of Theorem 1.5. As before, it suffices to consider the manifold $\widetilde{M}_{\mu, c}^{0}$ obtained by blowing up $M_{\mu}^{0}$ at a ball $B_{c}$ of capacity $c$. First, suppose that $c<\lambda$. We want to show that the group $\operatorname{Symp}\left(\widetilde{M}_{\mu, c}^{0}\right)$ is homotopy equivalent to the stabilizer of a point in $M_{\mu}^{0}$. By Lemma 1.2 above and Lemma 2.3 of [LP04], we know that $\operatorname{Symp}\left(\widetilde{M}_{\mu, c}^{0}\right)$ is homotopy equivalent to the group $\operatorname{Symp}^{\mathrm{U}(2)}\left(M_{\mu}^{0}, B_{c}\right)$ of those symplectomorphisms which act $\mathrm{U}(2)$-linearly in a neighborhood of $B_{c}$. Note that this group is included in the stabilizer of the center $p \in B_{c}$. Moreover, Theorem 1.3 implies that for any $c^{\prime} \in(0, c)$, the inclusion $i_{c, c^{\prime}}: \operatorname{Symp}^{\mathrm{U}(2)}\left(M_{\mu}^{0}, B_{c}\right) \hookrightarrow \operatorname{Symp}^{\mathrm{U}(2)}\left(M_{\mu}^{0}, B_{c^{\prime}}\right)$ is a homotopy equivalence. Furthermore, by Moser argument, every compact subset of the stabilizer $\operatorname{Symp}\left(M_{\mu}^{0}, p\right)$ is homotopic to a family of symplectomorphisms that act linearly on a ball of sufficiently small capacity centered at $p$. Therefore, the natural map

$$
\underset{\lim }{\longrightarrow} \operatorname{Symp}^{\mathrm{U}(2)}\left(M_{\mu}^{0}, B_{c}\right) \rightarrow \operatorname{Symp}\left(M_{\mu}^{0}, p\right)
$$

between the direct limit defined by the inclusions $\left\{i_{c, c^{\prime}}\right\}$ and the stabilizer is a (weak) homotopy equivalence.

When $\lambda \leqslant c$, the blow-up of $M_{\mu}^{0}$ at $B_{c}$ is symplectomorphic to the blow-up of a small ball $B_{1-c}$ in the $c$-related manifold $M_{\mu}^{0, c}=M_{\mu-c}^{1}$. The same argument as before shows that in this case $\operatorname{Symp}\left(\widetilde{M}_{\mu, c}^{0}\right) \simeq \operatorname{Symp}\left(M_{\mu-c}^{1}, p\right)$.

\subsection{The group $\operatorname{Symp}\left(\widetilde{M}_{\mu, c}^{1}, \Sigma\right)$ when $0<\mu<c<1$}

As explained in the Introduction, when $0<\mu<c<1$, the manifold $\widetilde{M}_{\mu, c}^{1}$ is conformally symplectomorphic to $\widetilde{M}_{\mu^{\prime}, c^{\prime}}^{0}$ where $\mu^{\prime}=1 /(\mu+1-c)$ and $c^{\prime}=(1-c) /(1+\mu-c)$. This identification takes the class $[\Sigma]$ to $B^{0}-E^{0}$ so that the space $\mathcal{J}_{\mu^{\prime}, c^{\prime}}^{0}$ is stratified according to the degeneracy types of curves representing $[\Sigma]$. The space $\mathcal{S}([\Sigma])$ of exceptional symplectic spheres in class $[\Sigma]$ is then homotopy equivalent to the open stratum $\mathcal{J}_{\mu^{\prime}, c^{\prime}, 0}^{0}$ which, in turn, is homotopy equivalent to $\operatorname{Symp}\left(\widetilde{M}_{\mu^{\prime}, c^{\prime}}\right) / T_{0}$. Looking at the fibration

$$
\operatorname{Symp}\left(\widetilde{M}_{\mu, c}^{1}, \Sigma\right) \simeq \operatorname{Symp}\left(\widetilde{M}_{\mu^{\prime}, c^{\prime}}^{0}, \Sigma\right) \rightarrow \operatorname{Symp}\left(\widetilde{M}_{\mu^{\prime}, c^{\prime}}^{0}\right) \rightarrow \mathcal{S}([\Sigma]) \simeq \operatorname{Symp}\left(\widetilde{M}_{\mu^{\prime}, c^{\prime}}^{0}\right) / T_{0},
$$

we conclude that $\operatorname{Symp}\left(\widetilde{M}_{\mu, c}^{1}, \Sigma\right) \simeq T_{0}$.

In order to compare $\operatorname{Symp}\left(\widetilde{M}_{\mu, c}^{1}, \Sigma\right)$ with the stabilizer of a point $p \in M_{\mu}^{1}$, we briefly describe a relative version of diagram (8). In fact, because we will use the same technique in our analysis of two disjoint balls in $\mathbb{C} P^{2}$, we consider more generally the group $\operatorname{Symp}(X, \mathcal{D})$ of symplectomorphisms which leave invariant each curve in the union $\mathcal{D}$ of $n$ disjoint, embedded, symplectic spheres representing some classes $D_{1}, \ldots, D_{n}$ in a symplectic 4-manifold $(X, \omega)$. Denote by $\mathcal{S}_{0}(\omega, \mathcal{D})$ the connected component of $\omega$ in the set of all symplectic forms cohomologous to $\omega$ and for which $\mathcal{D}$ is symplectic. Standard arguments involving the Mozer stability theorem in dimension 2, the extension of Hamiltonian isotopies, and the relative version of the Moser theorem imply that the identity component of the group $\operatorname{Diff}(X, \mathcal{D})$ (made of those diffeomorphisms sending each curve of $\mathcal{D}$ to itself) acts transitively on $\mathcal{S}_{0}(\omega, \mathcal{D})$. This action defines a fibration

$$
\operatorname{Symp}(X, \mathcal{D}) \cap \operatorname{Diff}_{0}(X, \mathcal{D}) \rightarrow \operatorname{Diff}_{0}(X, \mathcal{D}) \rightarrow \mathcal{S}_{0}(\omega, \mathcal{D})
$$

whose base is homotopy equivalent to the space $\mathcal{X}(\omega, \mathcal{D})$ of pairs $(\tau, J)$ such that $\tau \in \mathcal{S}_{0}(\omega, \mathcal{D}), J$ tames $\omega$, and $\mathcal{D}$ is $J$-holomorphic. As before, the projection on the second factor $\mathcal{X}(\omega, \mathcal{D}) \rightarrow \mathcal{A}(\omega, \mathcal{D})$ is a homotopy equivalence so that we can compare the symplectic stabilizer of $\mathcal{D}$ associated to 


\section{SYMPLECTOMORPHISMS AND EMBEDDINGS}

different symplectic forms through the following diagram.

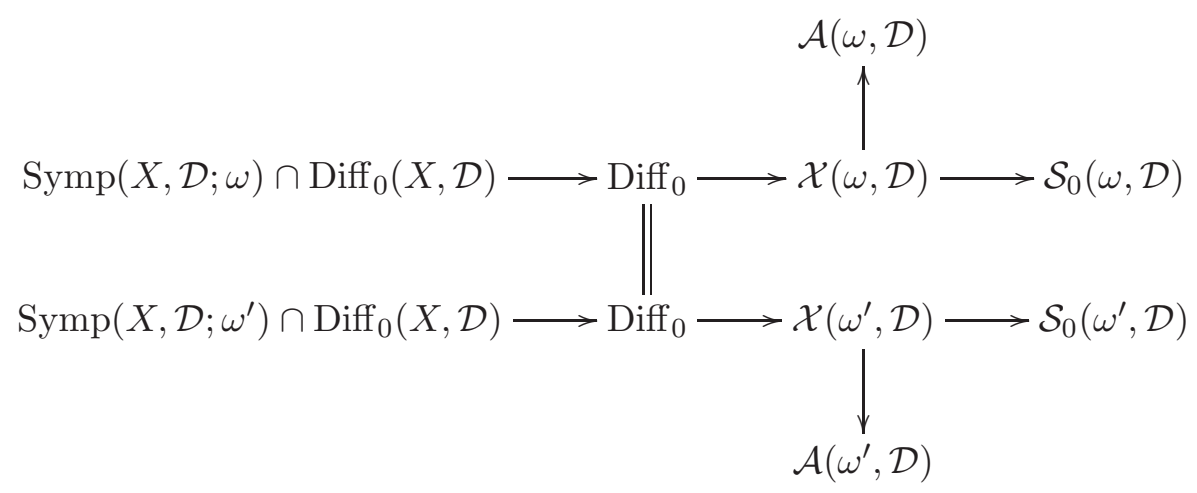

Proof of Theorem 1.6. In the case of the (connected) group $\operatorname{Symp}\left(\widetilde{M}_{\mu, c}^{1}, \Sigma\right)$ with $0<\mu<c<1$, the space $\mathcal{A}(\omega, \Sigma)$ belongs to the open stratum $\mathcal{A}_{\mu^{\prime}, c^{\prime}, 0} \subset \mathcal{A}_{\mu^{\prime}, c^{\prime}}$. Therefore, for each $J \in \mathcal{A}(\omega, \Sigma)$, there exist embedded $J$-holomorphic spheres in classes $F^{0}, B^{0}$, and $B^{0}+F^{0}-E^{0}$. Performing the inflation along theses curves (as in the proof of Theorem 1.3) shows that $\mathcal{A}(\omega, \Sigma)$ in independent of $\mu$ and $c$, so that we get a coherent family of homotopy equivalences $\operatorname{Symp}\left(\widetilde{M}_{\mu, c}^{1}, \Sigma\right) \rightarrow \operatorname{Symp}\left(\widetilde{M}_{\mu^{\prime \prime}, c^{\prime \prime}}, \Sigma\right)$ whenever $0<\mu^{\prime \prime}<c^{\prime \prime}<1$. Because the group $\operatorname{Symp}\left(M_{\mu}^{1}\right)$ is homotopy equivalent to U(2) when $\mu \leqslant 1$ (see Theorem 3.7 below), we can look at the direct limit of the groups $\operatorname{Symp}\left(\widetilde{M}_{\mu=c / 2, c}, \Sigma\right)$ as $c \rightarrow 0$ to conclude that $\operatorname{Symp}\left(\widetilde{M}_{\mu, c}^{1}, \Sigma\right)$ is homotopy equivalent to the stabilizer of a point in $M_{\mu}^{1}$ when $0<\mu<c<1$.

\subsection{Formal consequences}

The homotopy type of the groups $\operatorname{Symp}\left(M_{\mu}^{i}\right)$ was first investigated by Gromov in his celebrated paper [Gro85] in which he proved the following results using pseudo-holomorphic techniques and cut-and-paste arguments.

TheOREM 3.7 (see Gromov [Gro85]).

(i) When $\mu=1$, the symplectomorphism group of the manifold $M_{\mu}^{0}$ retracts onto its subgroup $(\mathrm{SO}(3) \times \mathrm{SO}(3)) \ltimes \mathbb{Z}_{2}$ of Kählerian isometries.

(ii) When $0<\mu \leqslant 1$, the symplectomorphism group of the manifold $M_{\mu}^{1}$ retracts onto its subgroup $\mathrm{U}(2)$ of Kählerian isometries.

Later, Abreu [Abr98] and then Abreu and McDuff [AM00] extended Gromov's techniques and computed the rational cohomology ring $H^{*}\left(\operatorname{Symp}\left(M_{\mu}^{i}\right) ; \mathbb{Q}\right)$. The rational cohomology ring $H^{*}\left(\operatorname{BSymp}\left(M_{\mu}^{i}\right) ; \mathbb{Q}\right)$ was computed by Abreu, Granja and Kitchloo [AGK06]. Their results can be summarized as follows.

Theorem 3.8 (see [AM00]). When $\mu>1$, the rational cohomology ring of the group $\operatorname{Symp}\left(M_{\mu}^{i}\right)$ is isomorphic to the product $\Lambda(t, x, y) \otimes \mathbb{Q}\left[w_{\ell}\right]$ with generators $t, x, y$, and $w_{\ell}$ of degree $1,3,3$, and $4 \ell+2 i$. Therefore, the rational homotopy groups of $\operatorname{Symp}\left(M_{\mu}^{i}\right)$ vanish in all dimensions except in dimensions 1,3 , and $4 \ell+2 i$, in which cases we have $\pi_{1}=\mathbb{Q}, \pi_{3}=\mathbb{Q}^{2}$, and $\pi_{4 \ell+2 i}=\mathbb{Q}$.

Theorem 3.9 (see [AM00] and [AGK06]). When $\mu>1$, the rational cohomology ring of the classifying space $\operatorname{BSymp}\left(M_{\mu}^{i}\right)$ is isomorphic to

$$
\mathbb{Q}[T, X, Y] / R_{\ell}
$$




\section{Pinsonnault}

where $R_{\ell}$ is the ideal generated by

$$
\begin{array}{ll}
T\left(T^{2}+X-Y\right)\left(T^{2}+16 X-4 Y\right) \cdots\left(T^{2}+\ell^{4} X-\ell^{2} Y\right) & \text { in the case } i=0, \\
\left(T^{2}+X-Y\right)\left(T^{2}+3^{4} X-3^{2} Y\right) \cdots\left(T^{2}+(2 \ell+1)^{4} X-(2 \ell+1)^{2} Y\right) & \text { in the case } i=1,
\end{array}
$$

and where the generators $T, X$, and $Y$ are of degree 2, 4, and 4 .

Combining these results with Theorem 1.5, we immediately get the following corollaries.

Corollary 3.10. Let $\widetilde{M}_{\mu, c}^{i}$ be the symplectic blow-up of $M_{\mu}^{i}$ at a ball of capacity $c \in(0,1)$ and suppose that $\mu>1$. Then, the following hold.

(1) When $c<\lambda$, the non-trivial rational homotopy groups of the topological group $\operatorname{Symp}\left(\widetilde{M}_{\mu, c}^{i}\right)$ are $\pi_{1} \simeq \mathbb{Q}^{3}$ and $\pi_{4 \ell+2 i} \simeq \mathbb{Q}$.

(2) When $\lambda \leqslant c$, the non-trivial rational homotopy groups of the topological group $\operatorname{Symp}\left(\widetilde{M}_{\mu, c}^{i}\right)$ are $\pi_{1} \simeq \mathbb{Q}^{3}$ and $\pi_{4 \ell+2 i-2} \simeq \mathbb{Q}$.

Proof. The rational homotopy groups of $\operatorname{Symp}\left(\widetilde{M}_{\mu, c}^{i}\right)$ are easily computed by combining Theorem 1.5 with Theorem 3.8. Indeed, suppose we blow up a small ball in $M_{\mu}^{0}$, where $\mu=\ell+\lambda>1$. Then we have a homotopy fibration

$$
\operatorname{Symp}\left(\widetilde{M}_{\mu, c}^{0}\right) \rightarrow \operatorname{Symp}\left(M_{\mu}^{0}\right) \rightarrow S^{2} \times S^{2}
$$

which gives an exact homotopy sequence whose only non-trivial terms are

$$
0 \rightarrow \pi_{4 \ell}\left(\operatorname{Symp}\left(\widetilde{M}_{\mu, c}^{0}\right)\right) \rightarrow \mathbb{Q} \rightarrow 0
$$

and

$$
\begin{aligned}
0 \rightarrow \pi_{3}\left(\operatorname{Symp}\left(\widetilde{M}_{\mu, c}^{0}\right)\right) & \rightarrow \mathbb{Q}^{2} \rightarrow \mathbb{Q}^{2} \rightarrow \pi_{2}\left(\operatorname{Symp}\left(\widetilde{M}_{\mu, c}^{0}\right)\right) \\
& \rightarrow 0 \rightarrow \mathbb{Q}^{2} \rightarrow \pi_{1}\left(\operatorname{Symp}\left(\widetilde{M}_{\mu, c}^{0}\right)\right) \rightarrow \mathbb{Q} \rightarrow 0 .
\end{aligned}
$$

In these exact sequences, the only problem is to understand the map

$$
\partial: \mathbb{Q}^{2} \simeq \pi_{3}\left(\operatorname{Symp}\left(M_{\mu}^{0}\right)\right) \rightarrow \pi_{2}\left(S^{2} \times S^{2}\right) \simeq \mathbb{Q}^{2} .
$$

But from the work of Abreu and McDuff, the group $\pi_{3}\left(M_{\mu}^{0}\right) \otimes \mathbb{Q}$ is identified with $\pi_{3}(\operatorname{SO}(3) \times$ $\mathrm{SO}(3)) \otimes \mathbb{Q}$ under the isometric action of $\mathrm{SO}(3) \times \mathrm{SO}(3)$ on $S^{2} \times S^{2}$. Therefore, the map $\partial$ is an isomorphism and both $\pi_{3}\left(\operatorname{Symp}\left(\widetilde{M}_{\mu, c}^{0}\right)\right)$ and $\pi_{2}\left(\operatorname{Symp}\left(\widetilde{M}_{\mu, c}^{0}\right)\right)$ are trivial.

When we blow up a ball of capacity $c \geqslant \lambda$ in $M_{\mu}^{0}$, the group $\operatorname{Symp}\left(\widetilde{M}_{\mu, c}^{0}\right)$ is homotopy equivalent to the stabilizer of a point in $\tilde{M}_{\mu-c}^{1}$. Since $\lfloor\mu-c\rfloor=\lfloor\ell+\lambda-c\rfloor=\ell-1$, the only non-trivial homotopy $\operatorname{groups}$ of $\operatorname{Symp}\left(\tilde{M}_{\mu-c}^{1}\right)$ are in degree 1,3 , and $4(\ell-1)+2=4 \ell-2$. When $\ell \geqslant 2$, the homotopy fibration

$$
\operatorname{Symp}\left(\widetilde{M}_{\mu, c}^{0}\right) \rightarrow \operatorname{Symp}\left(\tilde{M}_{\mu-c}^{1}\right) \rightarrow S^{2} \tilde{\times} S^{2}
$$

gives rise to an exact homotopy sequence whose only non-trivial terms are

$$
0 \rightarrow \pi_{4 \ell-2}\left(\operatorname{Symp}\left(\widetilde{M}_{\mu, c}^{0}\right)\right) \rightarrow \mathbb{Q} \rightarrow 0
$$

and

$$
\begin{aligned}
0 \rightarrow \pi_{3}\left(\operatorname{Symp}\left(\widetilde{M}_{\mu, c}^{0}\right)\right) & \rightarrow \mathbb{Q}^{2} \rightarrow \mathbb{Q}^{2} \rightarrow \pi_{2}\left(\operatorname{Symp}\left(\widetilde{M}_{\mu, c}^{0}\right)\right) \\
& \rightarrow 0 \rightarrow \mathbb{Q}^{2} \rightarrow \pi_{1}\left(\operatorname{Symp}\left(\widetilde{M}_{\mu, c}^{0}\right)\right) \rightarrow \mathbb{Q} \rightarrow 0 .
\end{aligned}
$$

When $\ell=1$, the exact homotopy sequence reduces to

$$
\begin{aligned}
0 \rightarrow \pi_{3}\left(\operatorname{Symp}\left(\widetilde{M}_{\mu, c}^{0}\right)\right) & \rightarrow \mathbb{Q}^{2} \rightarrow \mathbb{Q}^{2} \rightarrow \pi_{2}\left(\operatorname{Symp}\left(\widetilde{M}_{\mu, c}^{0}\right)\right) \\
& \rightarrow \pi_{2}\left(\operatorname{Symp}\left(M_{\mu-c}^{1}\right) \stackrel{0}{\rightarrow} \mathbb{Q}^{2} \rightarrow \pi_{1}\left(\operatorname{Symp}\left(\widetilde{M}_{\mu, c}^{0}\right)\right) \rightarrow \mathbb{Q} \rightarrow 0 .\right.
\end{aligned}
$$




\section{SympleCtOMORPHISMS AND EMBEDDINGS}

In both cases, Lemma 2.11 in Abreu and McDuff [AM00] implies that the homotopy group $\pi_{3}\left(\operatorname{Symp}\left(\tilde{M}_{\mu-c}^{1}\right)\right)$ is isomorphic to $\pi_{3}(\mathcal{D}) \otimes \mathbb{Q} \simeq \mathbb{Q}^{2}$, where $\mathcal{D}$ is the group of orientation-preserving fiberwise diffeomorphisms of $S^{2} \tilde{\times} S^{2}$, and that the connecting map $\partial: \pi_{3}\left(\operatorname{Symp}\left(\tilde{M}_{\mu-c}^{1}\right)\right) \rightarrow$ $\pi_{2}\left(S^{2} \tilde{\times} S^{2}\right) \simeq \mathbb{Q}^{2}$ is an isomorphism. Therefore, $\pi_{3}\left(\operatorname{Symp}\left(\widetilde{M}_{\mu, c}^{0}\right)\right)$ is trivial while $\pi_{2}\left(\operatorname{Symp}\left(\widetilde{M}_{\mu, c}^{0}\right)\right) \simeq$ $\pi_{2}\left(\operatorname{Symp}\left(M_{\mu-c}^{1}\right)\right)$.

Note that the vector space $\pi_{*}\left(\operatorname{Symp}\left(\widetilde{M}_{\mu, c}^{i}\right)\right) \otimes \mathbb{Q}$ has three topological generators of degree 1 which do not depend on the cohomology class of the symplectic form, and one symplectic generator whose degree changes precisely when $\mu$ crosses an integer or when the capacity $c$ reaches the critical value $\lambda$. The topological generators are robust in the sense that their images in $\pi_{*}($ Diff $) \otimes \mathbb{Q}$ generate a three-dimensional subspace, while the symplectic generator is fragile, that is, it does not survive the inclusion Symp $\hookrightarrow$ Diff.

Corollary 3.11. Let $\widetilde{M}_{\mu, c}^{i}$ be the symplectic blow-up of $M_{\mu}^{i}$ at a ball of capacity $c \in(0,1)$. In the case $i=0$, suppose that $\mu>1$, while in the case $i=1$ suppose that $c \leqslant \mu$. Then, the following hold.

(1) When $c<\lambda$, the rational cohomology ring of the group $\operatorname{Symp}\left(\widetilde{M}_{\mu, c}^{i}\right)$ is isomorphic to the product $\Lambda\left(\alpha_{1}, \alpha_{2}, \alpha_{3}\right) \otimes \mathbb{Q}[\epsilon]$ with generators $\alpha_{1}, \alpha_{2}, \alpha_{3}$, and $\epsilon$ of degree $1,1,1$, and $4 \ell+2 i$.

(2) When $\lambda \leqslant c$, the rational cohomology ring of the group $\operatorname{Symp}\left(\widetilde{M}_{\mu, c}^{i}\right)$ is isomorphic to the product $\Lambda\left(\alpha_{1}, \alpha_{2}, \alpha_{3}\right) \otimes \mathbb{Q}[\epsilon]$ with generators $\alpha_{1}, \alpha_{2}, \alpha_{3}$, and $\epsilon$ of degree $1,1,1$, and $4 \ell+2 i-2$.

Proof. Let $G$ be a connected topological group. The Samelson product $\langle\cdot, \cdot\rangle: \pi_{p}(G) \otimes \pi_{q}(G) \rightarrow$ $\pi_{p+q}(G)$ defined by the commutator map

$$
\begin{aligned}
\langle\alpha, \beta\rangle: S^{p} \wedge S^{q} & \rightarrow G \\
(s, t) & \mapsto \alpha(s) \beta(t) \alpha^{-1}(s) \beta^{-1}(t)
\end{aligned}
$$

gives the rational homotopy of $G$ the structure of a graded Lie algebra. The rational homology $H_{*}(G ; \mathbb{Q})$ is a connected and co-commutative graded Hopf algebra when equipped with the Pontrjagin product induced by the multiplication map. The Milnor-Moore theorem states that the Hurewicz homomorphism $\pi_{*}(G) \otimes \mathbb{Q} \rightarrow H_{*}(G ; \mathbb{Q})$ induces an isomorphism of Hopf algebras between the universal enveloping algebra $\mathcal{U}\left(\pi_{*}(G) \otimes \mathbb{Q}\right)$ and $H_{*}(G ; \mathbb{Q})$. In particular, it implies that $H_{*}(G ; \mathbb{Q})$ is always generated by its spherical classes.

In our situation, Corollary 3.10 implies that the rational homology of $\operatorname{Symp}\left(\widetilde{M}_{\mu, c}^{i}\right)$ is of finite type. Consequently, the rational cohomology $H^{*}\left(\operatorname{Symp}\left(\widetilde{M}_{\mu, c}^{i} ; \mathbb{Q}\right)\right)$ is a commutative Hopf algebra dual to the homology Hopf algebra. By the Borel classification of commutative Hopf algebra over fields of characteristic 0 , this implies that

$$
H^{*}\left(\operatorname{Symp}\left(\widetilde{M}_{\mu, c}^{i} ; \mathbb{Q}\right)\right) \simeq \Lambda\left(\alpha_{1}, \alpha_{2}, \alpha_{3}\right) \otimes \mathbb{Q}[\epsilon],
$$

where $\alpha_{1}, \alpha_{2}, \alpha_{3}$ are three generators of degree 1 , and $\epsilon$ is a generator of degree $4 \ell+2 i$ or $4 \ell+2 i-2$ depending on whether $c<\lambda$ or $c \geqslant \lambda$.

Since the group $\operatorname{Symp}\left(M_{\mu}^{i}\right)$ acts transitively on $M_{\mu}^{i}$, the classifying space of the isotropy group $\operatorname{Symp}\left(M_{\mu}^{i}, p\right)$ can be understood from the homotopy fibration

$$
M_{\mu}^{i} \rightarrow B \operatorname{Symp}\left(M_{\mu}^{i}, p\right) \rightarrow B \operatorname{Symp}\left(M_{\mu}^{i}\right) .
$$

This leads to the following two corollaries.

Corollary 3.12. Let $\widetilde{M}_{\mu, c}^{i}$ be as in Corollary 3.10. Then, the following hold.

(1) When $c<\lambda$, the rational cohomology module of the classifying space $\operatorname{BSymp}\left(\widetilde{M}_{\mu, c}^{i}\right)$ is isomorphic to $H^{*}\left(\operatorname{BSymp}\left(M_{\mu}^{i}\right)\right) \otimes H^{*}\left(M_{\mu}^{i}\right)$. 


\section{Pinsonnault}

(2) When $\lambda \leqslant c$, the rational cohomology module of the classifying space $\operatorname{BSymp}\left(\widetilde{M}_{\mu, c}^{i}\right)$ is isomorphic to $H^{*}\left(\operatorname{BSymp}\left(M_{\mu}^{i, c}\right)\right) \otimes H^{*}\left(M_{\mu}^{i, c}\right)$.

Proof. By Theorem 3.9, the rational cohomology rings of both $M_{\mu}^{i}$ and $B \operatorname{Symp}\left(M_{\mu}^{i}\right)$ are supported in even degrees. Consequently, the spectral sequence associated to this fibration collapses at the second stage $E_{2}^{p, q}$. Therefore,

$$
H^{*}\left(B \operatorname{Symp}\left(M_{\mu}^{i}, p\right) ; \mathbb{Q}\right) \simeq H^{*}\left(M_{\mu}^{i} ; \mathbb{Q}\right) \otimes H^{*}\left(B \operatorname{Symp}\left(M_{\mu}^{i}\right) ; \mathbb{Q}\right)
$$

and the result follows from Theorem 1.5.

Corollary 3.13. The Pontrjagin product on $H_{*}\left(\operatorname{Symp}\left(\widetilde{M}_{\mu, c}^{0}\right) ; \mathbb{Q}\right)$ is (graded) commutative and the Samelson product on $\pi_{*}\left(\operatorname{Symp}\left(\widetilde{M}_{\mu, c}^{0}\right)\right) \otimes \mathbb{Q}$ is trivial, except when $1<\mu<2$ and $\lambda \leqslant c$ in which case the Samelson products $\left\langle\alpha_{1}, \alpha_{3}\right\rangle=-\left\langle\alpha_{2}, \alpha_{3}\right\rangle$ are non-zero multiples of $\epsilon \in \pi_{2}\left(\operatorname{Symp}\left(\widetilde{M}_{\mu, c}^{0}\right)\right) \otimes \mathbb{Q}$.

Proof. (See [LP04] for an alternative proof.) For dimensional reasons, the Samelson products defined on $\pi_{*}\left(\operatorname{Symp}\left(\widetilde{M}_{\mu, c}^{0}\right)\right) \otimes \mathbb{Q}$ are all trivial except possibly when $1<\mu<2$ and $\lambda \leqslant c$. In that case, the jumping generator $\epsilon$ has degree 2 and hence the products $\left\langle\alpha_{i}, \alpha_{j}\right\rangle$ might be non-zero. By [LP04, Proposition 4.7], we can choose the classes $\alpha_{1}, \alpha_{2}, \alpha_{3}$ such that $\alpha_{1}, \alpha_{2}$ correspond to lifts (to the blow-up) of the generators of the standard Kählerian (and Hamiltonian) $T^{2}$-action on the Hirzebruch surface $W^{1}$, while $\alpha_{1}+\alpha_{2}, \alpha_{3}$ correspond to lifts of the generators of the Kählerian $T^{2}$-action on $W^{2}$. Consequently, $\left\langle\alpha_{1}, \alpha_{2}\right\rangle=0$ while $\left\langle\alpha_{1}, \alpha_{3}\right\rangle=-\left\langle\alpha_{2}, \alpha_{3}\right\rangle$.

Now, to understand the product $\left\langle\alpha_{1}, \alpha_{3}\right\rangle$, we use the fact that it corresponds (up to sign) to a rational Whitehead product in the homotopy of the classifying space $B \operatorname{Symp}\left(\widetilde{M}_{\mu, c}^{0}\right)$. Since the non-trivial homotopy groups of $B \operatorname{Symp}\left(\widetilde{M}_{\mu, c}^{0}\right)$ are $\pi_{2} \simeq \mathbb{Q}^{3}$ and $\pi_{3} \simeq \mathbb{Q}$, the minimal model of $B \operatorname{Symp}\left(\widetilde{M}_{\mu, c}^{0}\right)$ has only four generators $a_{2}, b_{2}, c_{2}, e_{3}$ (here the index is the degree). By the Hurewicz theorem, $\pi_{2} \simeq H_{2} \simeq H^{2}$, which implies that $a_{2}, b_{2}, c_{2}$ are cocycles. Moreover, by Corollary 3.12 we have

$$
H_{k}\left(B \operatorname{Symp}\left(\widetilde{M}_{\mu, c}^{0}\right)\right) \simeq \begin{cases}0, & \text { if } k \text { is odd } \\ \mathbb{Q}^{k+1}, & \text { if } k \text { is even }\end{cases}
$$

and this implies that $e_{3}$ cannot be a cocycle. Thus

$$
d e_{3}=\text { a linear combination of } a_{2}^{2}, b_{2}^{2}, c_{2}^{2}, a_{2} b_{2}, a_{2} c_{2} \text {, and } b_{2} c_{2} .
$$

By the description of the rational Whitehead products given by Andrews and Arkowitz [AA78], this implies that the Whitehead product map

$$
[-,-]: \pi_{2}\left(B \operatorname{Symp}\left(\widetilde{M}_{\mu, c}^{0}\right)\right) \otimes \pi_{2}\left(B \operatorname{Symp}\left(\widetilde{M}_{\mu, c}^{0}\right)\right) \rightarrow \pi_{3}\left(B \operatorname{Symp}\left(\widetilde{M}_{\mu, c}^{0}\right)\right)
$$

is non-trivial or, after desuspension, that the Samelson product map

$$
\langle-,-\rangle: \pi_{1}\left(\operatorname{Symp}\left(\widetilde{M}_{\mu, c}^{0}\right)\right) \otimes \pi_{1}\left(\operatorname{Symp}\left(\widetilde{M}_{\mu, c}^{0}\right)\right) \rightarrow \pi_{2}\left(\operatorname{Symp}\left(\widetilde{M}_{\mu, c}^{0}\right)\right)
$$

is non-trivial, that is, the product $\left\langle\alpha_{1}, \alpha_{3}\right\rangle$ is a non-zero multiple of the jumping generator $\epsilon$.

\subsection{The integral homology $H^{*}\left(\operatorname{Symp}\left(\tilde{M}_{\mu, c}^{0}\right) ; \mathbb{Z}\right)$ when $\mu \in(1,2)$ and $\lambda \leqslant c$}

We now use the description of the stratification of the space of tamed almost complex structures $\tilde{\mathcal{J}}_{\mu, c}=\mathcal{J}\left(\widetilde{M}_{\mu, c}^{0}\right)$ given in $\S 2.4$ to prove that $H^{*}\left(\operatorname{Symp}\left(\tilde{M}_{\mu, c}^{0}\right) ; \mathbb{Z}\right)$ has no torsion.

For that range of parameters, the contractible space $\tilde{\mathcal{J}}_{\mu, c}=\tilde{\mathcal{J}}_{01}$ is the union of the open, dense, and connected stratum $\tilde{\mathcal{J}}_{0}$ with the codimension 2 , co-oriented, closed submanifold $\tilde{\mathcal{J}}_{1}$. Hence, a tubular neighborhood $\mathcal{N}$ of $\tilde{\mathcal{J}}_{1}$ is homeomorphic to an oriented disc bundle $D^{2} \rightarrow \mathcal{N} \rightarrow \tilde{\mathcal{J}}_{1}$. Let $\mathbb{F}$ denote either $\mathbb{Q}$ or a finite field $\mathbb{F}_{p}$ for $p \geqslant 2$ prime. 


\section{SympleCtOMORPHISMS AND EMBEDDINGS}

Lemma 3.14. There is an isomorphism $H_{i+1}\left(\tilde{\mathcal{J}}_{0} ; \mathbb{F}\right) \simeq H_{i}\left(\tilde{\mathcal{J}}_{1} ; \mathbb{F}\right)$, for all $i \geqslant 0$.

Proof. Since the space $\tilde{\mathcal{J}}_{01}$ is contractible, the long exact sequence of the pair $\left(\tilde{\mathcal{J}}_{01}, \tilde{\mathcal{J}}_{0}\right)$ shows that the groups $H_{i+2}\left(\tilde{\mathcal{J}}_{01}, \tilde{\mathcal{J}}_{0}\right)$ and $H_{i+1}\left(\tilde{\mathcal{J}}_{0}\right)$ are isomorphic. Write $\xi$ for the intersection $\tilde{\mathcal{J}}_{0} \cap \mathcal{N}$. Excising $\tilde{\mathcal{J}}_{01}-\mathcal{N}$ from $\left(\tilde{\mathcal{J}}_{01}, \tilde{\mathcal{J}}_{0}\right)$, we see that $H_{i+2}\left(\tilde{\mathcal{J}}_{01}, \tilde{\mathcal{J}}_{0}\right) \simeq H_{i+2}(\mathcal{N}, \xi)$. By the Thom isomorphism theorem, there is an isomorphism $\left.H_{i+2}(\mathcal{N}), \xi\right) \simeq H_{i}\left(\tilde{\mathcal{J}}_{1}\right)$ for all $i \geqslant 0$.

Each stratum $\tilde{\mathcal{J}}_{i}$ is homotopy equivalent to the quotient $\operatorname{Symp}\left(\widetilde{M}_{\mu, c}^{0}\right) / \tilde{T}_{i}$ and we have two homotopy fibrations $\tilde{T}_{i} \rightarrow \operatorname{Symp}\left(\widetilde{M}_{\mu, c}^{0}\right) \rightarrow \tilde{\mathcal{J}}_{i}$.

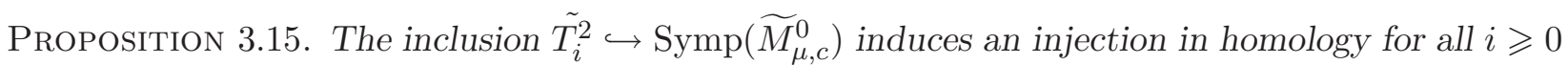
and $\mu \geqslant 1$, and for any coefficient field $\mathbb{F}$.

Proof. First note that the inclusion $\tilde{T}_{i}^{2} \hookrightarrow \operatorname{Symp}\left(\widetilde{M}_{\mu, c}^{0}\right)$ induces a morphism of Hopf algebras. Since $H_{*}\left(\tilde{T}_{i}^{2} ; \mathbb{F}\right)$ is primitively generated and commutative, it is enough to show that its submodule of primitive elements, which is simply $H_{1}\left(\tilde{T}_{i}^{2} ; \mathbb{F}\right)$, is mapped injectively into $H_{1}\left(\operatorname{Symp}\left(\widetilde{M}_{\mu, c}^{0}\right) ; \mathbb{F}\right)$.

The same proof as in Lemma 1.2 shows that the $\operatorname{space} \operatorname{Symp}\left(\widetilde{M}_{\mu, c}^{0}\right)$ is homotopy equivalent to $\operatorname{Symp}\left(\widetilde{M}_{\mu, c}^{0}, E \cup(F-E)\right)$. One therefore gets a map from $\operatorname{Symp}\left(\widetilde{M}_{\mu, c}^{0}\right) \rightarrow \operatorname{Symp}\left(\widetilde{M}_{\mu, c}^{0}, E \cup(F-E)\right) \rightarrow$ $\mathcal{E}\left(W_{i}, F_{i}, *\right)$, the space of homotopy self-equivalences of $W_{i}$ that preserve a fiber $F_{i}$, and $*$, the point of intersection between $F_{i}$ and the section of self-intersection $-i$. Here the last map is the one induced by blowing down an exceptional curve in class $E$ when $i=1$ and $F-E$ when $i=2$. Consider the maps

$$
\mathcal{E}\left(W_{i}, F_{i}, *\right) \rightarrow \mathcal{E}\left(F_{i}, *\right)
$$

given by the restriction to the fiber $F_{i}$ and

$$
\mathcal{E}\left(W_{i}, F_{i}, *\right) \rightarrow \mathcal{E}\left(S^{2}, *\right)
$$

given by first embedding $S^{2}$ in $W_{i}$ as the zero section, applying a homotopy equivalence, and then projecting back to the base of the Hirzebruch fibration. These two maps show that the images in $\pi_{1}\left(\operatorname{Symp}\left(\widetilde{M}_{\mu, c}^{0}\right)\right)$ of the two generators of $\pi_{1}\left(\tilde{T}_{i}\right)$ have infinite order and are independent. Since $\operatorname{Symp}\left(\widetilde{M}_{\mu, c}^{0}\right)$ is a topological group, $\pi_{1}\left(\operatorname{Symp}\left(\widetilde{M}_{\mu, c}^{0}\right)\right)=H_{1}\left(\operatorname{Symp}\left(\widetilde{M}_{\mu, c}^{0}\right) ; \mathbb{Z}\right)$ and the proposition follows readily.

Corollary 3.16. There is an isomorphism of graded vector spaces

$$
H_{*}\left(\operatorname{Symp}\left(\tilde{M}_{\mu, c}\right) ; \mathbb{F}\right) \simeq H_{*}\left(\tilde{\mathcal{J}}_{i} ; \mathbb{F}\right) \otimes H_{*}\left(\tilde{T}_{i}^{2} ; \mathbb{F}\right)
$$

and an isomorphism of graded algebras

$$
H^{*}\left(\operatorname{Symp}\left(\tilde{M}_{\mu, c}\right) ; \mathbb{F}\right) \simeq H^{*}\left(\tilde{\mathcal{J}}_{i} ; \mathbb{F}\right) \otimes H^{*}\left(\tilde{T}_{i}^{2} ; \mathbb{F}\right) .
$$

Consequently, $H^{*}\left(\tilde{\mathcal{J}}_{0} ; \mathbb{F}\right) \simeq H^{*}\left(\tilde{\mathcal{J}}_{1} ; \mathbb{F}\right)$.

Proof. This follows from the Leray-Hirsh theorem applied to the homotopy fibrations

$$
\tilde{T}_{i} \rightarrow \operatorname{Symp}\left(\widetilde{M}_{\mu, c}^{0}\right) \rightarrow \tilde{\mathcal{J}}_{i}
$$

and from the fact that $H^{*}\left(\tilde{T}_{i}^{2} ; \mathbb{F}\right)$ is a free algebra.

Proposition 3.17. When $1<\mu<2$ and $\lambda \leqslant c, H_{*}\left(\operatorname{Symp}\left(\widetilde{M}_{\mu, c}^{0}\right) ; \mathbb{Z}\right)$ has no torsion.

Proof. Combining Lemma 3.14 with Corollary 3.16 and using the fact that $\tilde{\mathcal{J}}_{i}$ is connected, we get $H^{q}\left(\tilde{\mathcal{J}}_{i} ; \mathbb{F}\right) \simeq \mathbb{F}$, for all $q \geqslant 0$. Putting this back into the isomorphism

$$
H_{*}\left(\operatorname{Symp}\left(\tilde{M}_{\mu, c}\right) ; \mathbb{F}\right) \simeq H_{*}\left(\tilde{\mathcal{J}}_{i} ; \mathbb{F}\right) \otimes H_{*}\left(\tilde{T}_{i}^{2} ; \mathbb{F}\right),
$$




\section{Pinsonnault}

this shows that the homology $H_{*}\left(\operatorname{Symp}\left(\tilde{M}_{\mu, c}\right) ; \mathbb{F}\right)$ is the tensor product $H_{*}\left(\operatorname{Symp}\left(\tilde{M}_{\mu, c}\right) ; \mathbb{Z}\right)_{\text {free }} \otimes \mathbb{F}$ and this implies that the integral homology (and cohomology) has no torsion.

From Proposition 3.6, we get the following result.

Corollary 3.18. For all $\mu>1$ and $c \in(0,1), \pi_{1}\left(\operatorname{Symp}\left(\widetilde{M}_{\mu, c}^{0}\right)\right) \simeq \mathbb{Z} \oplus \mathbb{Z} \oplus \mathbb{Z}$.

Remark 3.19. When $\mu \in(1,2)$ and $\lambda \leqslant c$, it can be shown, using arguments similar to the ones used in Anjos [Anj02] and Anjos and Granja [AG04], that $\operatorname{Symp}\left(\widetilde{M}_{\mu, c}^{0}\right)$ is homotopy equivalent (as a space) to $\Omega S^{3} \times S^{1} \times S^{1} \times S^{1}$.

\section{Spaces of embeddings}

We now investigate the homotopy type of the space of symplectic embeddings $\Im \operatorname{Emb}\left(B_{c}, M_{\mu}^{i}\right)$.

Proof of Theorem 1.7. Recall that McDuff [McD96] showed that the embedding spaces $\Im \operatorname{Emb}\left(B_{c}, M_{\mu}^{i}\right)$ are always connected. Consequently, we have a homotopy fibration

$$
\operatorname{Symp}\left(\widetilde{M}_{\mu, c}^{i}, \Sigma\right) \rightarrow \operatorname{Symp}\left(M_{\mu}^{i}\right) \rightarrow \Im \operatorname{Emb}\left(B_{c}, M_{\mu}^{i}\right) .
$$

When $c<\lambda$, Theorem 1.5 states that the fiber $\operatorname{Symp}\left(\widetilde{M}_{\mu, c}^{i}, \Sigma\right)$ is homotopy equivalent to the symplectic stabilizer of a point in $M_{\mu}^{i}$. Consequently, $\Im \operatorname{Emb}\left(B_{c}, M_{\mu}^{i}\right)$ has the same homotopy type as $M_{\mu}^{i}$.

When $\lambda \leqslant c<\mu$, the rational homotopy groups of $\operatorname{Symp}\left(\widetilde{M}_{\mu, c}^{i}, \Sigma\right)$ and $\operatorname{Symp}\left(M_{\mu}^{i}\right)$ are given in Corollary 3.10 and Theorem 3.8. The only ambiguous part in the rational homotopy sequence of the above fibration is

$$
\rightarrow 0 \rightarrow \pi_{2}(\Im \operatorname{Emb}) \rightarrow \pi_{1}\left(\operatorname{Symp}\left(\widetilde{M}_{\mu, c}^{0}\right)\right) \rightarrow \pi_{1}\left(\operatorname{Symp}\left(M_{\mu}^{0}\right)\right) \rightarrow \pi_{1}(\Im \operatorname{Emb}) \rightarrow 0 .
$$

But since the blow-up of the generator of the group $\pi_{1}\left(\operatorname{Symp}\left(M_{\mu}^{0}\right)\right) \simeq \mathbb{Q}$ is one of the generators of $\pi_{1}\left(\operatorname{Symp}\left(\widetilde{M}_{\mu, c}^{0}\right)\right) \simeq \mathbb{Q}^{3}$, the map $\pi_{1}\left(\operatorname{Symp}\left(\widetilde{M}_{\mu, c}^{0}\right)\right) \rightarrow \pi_{1}\left(\operatorname{Symp}\left(M_{\mu}^{0}\right)\right)$ is surjective and, therefore, $\pi_{1}(\Im \mathrm{Emb})=0$ and $\pi_{2}(\Im \mathrm{Emb}) \simeq \mathbb{Q}$. The rest of the computation of $\pi_{*}(\Im \mathrm{Emb})$ is straightforward.

Proof of Theorem 1.8. This follows directly from Theorem 1.6 applied to the fibration (15).

\subsection{Symplectic balls in $\mathbb{C} P^{2}$}

We now apply the relative framework of $\S 3.4$ to determine the homotopy type of the space of one or two disjoint symplectic balls in $\mathbb{C} P^{2}$. Recall that if we normalize $\mathbb{C} P^{2}$ so that the area of a line $L$ is 1 , then the embedding spaces $\operatorname{Emb}\left(B_{c}, \mathbb{C} P^{2}\right)$ and $\operatorname{Emb}\left(B_{c_{1}} \sqcup B_{c_{2}}, \mathbb{C} P^{2}\right)$ are non-empty if, and only if, $\delta \in(0,1)$ and $\delta_{1}+\delta_{2} \in(0,1)$; see [MP94]. By the work of McDuff [McD96], we know that these spaces are connected.

Proof of Theorem 1.10. We first consider the embedding of one ball in $\mathbb{C} P^{2}$. Let $\Sigma$ denote the exceptional divisor in $\widetilde{\mathbb{C} P}^{2}$ and consider the fibrations

$$
\operatorname{Symp}\left(\widetilde{\mathbb{C} P}^{2}, \Sigma\right) \rightarrow \operatorname{Symp}\left(\mathbb{C} P^{2}\right) \rightarrow \Im \operatorname{Emb}\left(B_{\delta}, \mathbb{C} P^{2}\right)
$$

and

$$
\operatorname{Symp}\left(\widetilde{\mathbb{C P}}^{2}, \Sigma\right) \cap \operatorname{Diff}_{0}\left(\widetilde{\mathbb{C P}}^{2}, \Sigma\right) \rightarrow \operatorname{Diff}_{0}\left(\mathbb{C} P^{2}, \Sigma\right) \rightarrow \mathcal{S}_{0}(\omega, \Sigma) \simeq \mathcal{A}(\omega, \Sigma) .
$$

The blow-up manifold $\widetilde{\mathbb{C P}}^{2}$ is conformally symplectomorphic to the non-trivial bundle $M_{\mu}^{1}$ with $\mu=\delta /(1+\delta) \in(0,1)$. The space $\mathcal{J}_{\mu}$ of all tame almost complex structures on $M_{\mu}^{1}$ has a stratification 


\section{SyMPLECTOMORPHISMS AND EMBEDDINGS}

induced by the degeneration type of the class $[\Sigma]=B^{1}$ (which, for $\mu>k \in \mathbb{N}$, can be represented by cusp-curves containing a component in the class $\left.B^{1}-k F^{1}\right)$. But for $\mu \in(0,1)$, the exceptional class $[\Sigma]$ is symplectically indecomposable. It follows that, for $\mu \in(0,1)$, this stratification is trivial and that $\operatorname{Symp}\left(\widetilde{\mathbb{C P}}^{2}, \Sigma\right) \simeq \operatorname{Symp}\left(M_{\mu}^{1}\right) \simeq \mathrm{U}(2)$. It also follows that, for all $J \in \mathcal{J}_{\mu}$, the classes $B^{1}$ and $F^{1}$ are represented by $J$-holomorphic embedded spheres. Inflating along these spheres shows that the space $\mathcal{A}(\omega, \Sigma)$ is independent of $\mu \in(0,1]$ so that we get a coherent family of homotopy equivalences $\operatorname{Symp}\left(M_{\mu}^{1}, \Sigma\right) \rightarrow \operatorname{Symp}\left(M_{\mu-\epsilon}^{1}, \Sigma\right)$. Looking at the limit when $\mu \rightarrow 0$ (or, equivalently, when $\delta \rightarrow 0)$, we conclude that $\operatorname{Symp}\left(M_{\mu}^{1}, \Sigma\right) \simeq \operatorname{Symp}\left(\widetilde{\mathbb{C P}}^{2}, \Sigma\right)$ is homotopy equivalent to the symplectic stabilizer of a point in $\mathbb{C} P^{2}$.

We now consider the embedding of two disjoint balls $\mathcal{B}:=B_{\delta_{1}} \sqcup B_{\delta_{2}}$ in $\mathbb{C} P^{2}$, where we suppose that $\delta_{1} \geqslant \delta_{2}$. Let denote by $\mathcal{D}=\Sigma_{1} \sqcup \Sigma_{2}$ the union of the exceptional divisors in the 2-fold blow-up $X_{\delta_{1}, \delta_{2}}$ and consider the fibrations

$$
\begin{gathered}
\operatorname{Symp}\left(X_{\delta_{1}, \delta_{2}}, \mathcal{D}\right) \rightarrow \operatorname{Symp}\left(\mathbb{C} P^{2}\right) \rightarrow \Im \operatorname{Emb}\left(\mathcal{B}, \mathbb{C} P^{2}\right) \\
\operatorname{Symp}\left(X_{\delta_{1}, \delta_{2}}, \mathcal{D}\right) \cap \operatorname{Diff}_{0}\left(X_{\delta_{1}, \delta_{2}}, \mathcal{D}\right) \rightarrow \operatorname{Diff}_{0}\left(X_{\delta_{1}, \delta_{2}}, \mathcal{D}\right) \rightarrow \mathcal{S}_{0}(\omega, \mathcal{D}) \simeq \mathcal{A}(\omega, \mathcal{D}) .
\end{gathered}
$$

This time, $X_{\delta_{1}, \delta_{2}}$ is conformally symplectomorphic to $\widetilde{M}_{\mu, c}^{0}$ with $\mu=\left(1-\delta_{2}\right) /\left(1-\delta_{1}\right) \geqslant 1$ and $c=\left(1-\delta_{1}-\delta_{2}\right) /\left(1-\delta_{1}\right) \in(0,1)$ via a diffeomorphism which sends the exceptional classes $[L]-\left[\Sigma_{1}\right]-\left[\Sigma_{2}\right],\left[\Sigma_{1}\right]$, and $\left[\Sigma_{2}\right]$ to, respectively, $E, B-E$, and $F-E$. In particular, $\mathcal{D}$ is a configuration of disjoint exceptional spheres in classes $B-E$ and $F-E$. Write $\mathcal{C}(\mathcal{D})$ for the set of all such configurations. Because, for any $J \in \mathcal{J}_{\mu, c}^{0}$, the class $F-E$ is represented by a unique embedded $J$-holomorphic sphere, $\mathcal{C}(\mathcal{D})$ is homotopy equivalent to the space $\mathcal{C}(B-E)$ of all exceptional symplectic spheres in class $B-E$. As explained before, $\mathcal{C}(B-E)$ is homotopy equivalent to the open stratum $\mathcal{J}_{\mu, c, 0}$ which is itself homotopy equivalent to the homogeneous space $\operatorname{Symp}\left(\widetilde{M}_{\mu, c}^{0}\right) / T_{0}$. It follows that $\operatorname{Symp}\left(X_{\delta_{1}, \delta_{2}}, \mathcal{D}\right) \simeq \operatorname{Symp}\left(\widetilde{M}_{\mu, c}^{0}, \mathcal{D}\right)$ is homotopy equivalent to the torus $T_{0}$. In particular, $\operatorname{Symp}\left(X_{\delta_{1}, \delta_{2}}, \mathcal{D}\right)$ is connected.

Because $\mathcal{A}(\omega, \mathcal{D})$ is contained in the open stratum of $\mathcal{A}_{\mu, c}$, it is independent of $\mu$ and $c$. As before, it follows that the homotopy type of the group $\operatorname{Symp}\left(X_{\delta_{1}, \delta_{2}}, \mathcal{D}\right)$ is independent of $\delta_{1}$ and $\delta_{2}$. Considering the limit as $\delta_{1}$ and $\delta_{2}=1-\delta_{1}-\epsilon$ go to zero shows that $\operatorname{Symp}\left(X_{\delta_{1}, \delta_{2}}, \mathcal{D}\right)$ is homotopy equivalent to the stabilizer of two points in $\mathbb{C} P^{2}$. This implies that $\Im \operatorname{Emb}\left(\mathcal{B}, \mathbb{C} P^{2}\right)$ is homotopy equivalent to the space of ordered configurations of two points in $\mathbb{C} P^{2}$.

\section{ACKNowledGements}

I am pleased to thank F. Lalonde, D. McDuff, and S. Anjos for helpful discussions, and also an anonymous referee for pointing out an error in my original treatment of Theorem 1.3 and for suggesting improvements to the exposition. Part of this work was carried out while I was a postdoctoral fellow at the Department of Mathematics of the University of Toronto and I take this opportunity to thank the staff and faculty for providing a stimulating environment. I also thank the Fields Institute where this work was completed.

\section{REFERENCES}

Abr98 M. Abreu, Topology of symplectomorphism groups of $S^{2} \times S^{2}$, Invent. Math. 131 (1998), 1-23.

AM00 M. Abreu and D. McDuff, Topology of symplectomorphism groups of rational ruled surfaces, J. Amer. Math. Soc. 13 (2000), 971-1009 (electronic).

AGK06 M. Abreu, G. Granja and N. Kitchloo, Symplectomorphism groups and compatible complex structures on rational ruled surfaces, Preprint (2006), arXiv math.SG/0610436. 


\section{SYMPLECTOMORPHISMS AND EMBEDDINGS}

AA78 P. Andrews and M. Arkowitz, Sullivan's minimal models and higher order Whitehead products, Canad. J. Math. 30 (1978), 961-982.

Anj02 S. Anjos, Homotopy type of symplectomorphism groups of $S^{2} \times S^{2}$, Geom. Topol. 6 (2002), 195-218 (electronic).

AG04 S. Anjos and G. Granja, Homotopy decomposition of a group of symplectomorphisms of $S^{2} \times S^{2}$, Topology 43 (2004), 599-618.

AL94 M. Audin and J. Lafontaine, Holomorphic curves in symplectic geometry, Progress in Mathematics, vol. 117 (Birkhäuser, Basel, 1994).

Del88 T. Delzant, Hamiltoniens périodiques et images convexes de l'application moment, Bull. Soc. Math. France 116 (1988), 315-339.

Gro85 M. Gromov, Pseudoholomorphic curves in symplectic manifolds, Invent. Math. 82 (1985), 307-347.

HLS97 H. Hofer, V. Lizan and J.-C. Sikorav, On genericity for holomorphic curves in four-dimensional almost-complex manifolds, J. Geom. Anal. 7 (1997), 149-159.

Kar99 Y. Karshon, Periodic Hamiltonian flows on four-dimensional manifolds, Mem. Amer. Math. Soc. 141 (1999).

Lal94 F. Lalonde, Isotopy of symplectic balls, Gromov's radius and the structure of ruled symplectic 4-manifolds, Math. Ann. 300 (1994), 273-296.

LM94 F. Lalonde and D. McDuff, J-curves and the classification of rational and ruled symplectic 4-manifolds, in Contact and symplectic geometry, Cambridge, 1994 (Cambridge University Press, Cambridge, 1994), 3-42.

LP04 F. Lalonde and M. Pinsonnault, The topology of the space of symplectic balls in rational 4-manifolds, Duke Math. J. 122 (2004), 347-397.

LL95 T. J. Li and A. Liu, General wall crossing formula, Math. Res. Lett. 2 (1995), 797-810.

LL99 T.-J. Li and A.-K. Liu, The equivalence between $S W$ and $G r$ in the case where $b^{+}=1$, Int. Math. Res. Not. 1999 (1999), 335-345.

McD90 D. McDuff, The structure of rational and ruled symplectic 4-manifolds, J. Amer. Math. Soc. 3 (1990), 679-712; Erratum, J. Amer. Math. Soc. 5 (1992), 987-988.

McD96 D. McDuff, From symplectic deformation to isotopy, in Topics in symplectic 4-manifolds, Irvine, CA, 1996 (International Press, Cambridge, MA, 1996), 85-99.

McD00 D. McDuff, Almost complex structures on $S^{2} \times S^{2}$, Duke Math. J. 101 (2000), 135-177.

McD03 D. McDuff, Symplectomorphism groups and almost complex structures, in Essays on geometry and related topics, vols 1, 2 (Enseignement Mathématique, Geneva, 2003), 527-556.

MP94 D. McDuff and L. Polterovich, Symplectic packings and algebraic geometry, Invent. Math. 115 (1994), 405-434.

MS94 D. McDuff and D. Salamon, J-holomorphic curves and quantum cohomology, University Lecture Series, vol. 6 (American Mathematical Society, Providence, RI, 1994).

Mil59 J. Milnor, On spaces having the homotopy type of CW-complex, Trans. Amer. Math. Soc. 90 (1959), $272-280$.

Sei03 P. Seidel, Lectures on four-dimensional Dehn twists, Preprint (2003), arXiv math.SG/0309012.

Tau96 C. H. Taubes, Counting pseudo-holomorphic submanifolds in dimension 4, J. Differential Geom. 44 (1996), 818-893.

Martin Pinsonnault pinsonnault@crm.umontreal.ca

Department of Mathematics, University of Toronto, Toronto, Canada, M5S 3G3

Current address: Fields Institute, Toronto, Canada, M5T 3J1 